OPEN ACCESS

Edited by:

Jing-wen Lin,

Sichuan University, China

Reviewed by:

Tracey Lamb,

The University of Utah, United States

Galadriel Hovel-Miner

George Washington University,

United States

*Correspondence:

Jian Wu

jian.wu2@nih.gov

Xin-Zhuan Su

xsu@niaid.nih.gov

${ }^{+}$These authors share first authorship

Specialty section: This article was submitted to

Parasite and Host,

a section of the journal

Frontiers in Cellular and

Infection Microbiology

Received: 13 August 2020

Accepted: 30 October 2020

Published: 02 December 2020

Citation:

He X, Xia L, Tumas KC, Wu J and Su X-Z (2020) Type I Interferons and

Malaria: A Double-Edge Sword Against a Complex Parasitic Disease. Front. Cell. Infect. Microbiol. 10:594621. doi: 10.3389/fcimb.2020.594621

\section{Type I Interferons and Malaria: A Double-Edge Sword Against a Complex Parasitic Disease}

\author{
Xiao $\mathrm{He}^{1 \dagger}$, Lu Xia ${ }^{1,2+}$, Keyla C. Tumas ${ }^{1}$, Jian $\mathrm{Wu}^{1 * t}$ and Xin-Zhuan Su${ }^{1 *}$ \\ ${ }^{1}$ Malaria Functional Genomics Section, Laboratory of Malaria and Vector Research, National Institute of Allergy and \\ Infectious Disease, National Institutes of Health, Bethesda, MD, United States, ${ }^{2}$ Center for Medical Genetics, School of \\ Life Sciences, Central South University, Changsha, China
}

Type I interferons (IFN-Is) are important cytokines playing critical roles in various infections, autoimmune diseases, and cancer. Studies have also shown that IFN-Is exhibit 'conflicting' roles in malaria parasite infections. Malaria parasites have a complex life cycle with multiple developing stages in two hosts. Both the liver and blood stages of malaria parasites in a vertebrate host stimulate IFN-I responses. IFN-Is have been shown to inhibit liver and blood stage development, to suppress $T$ cell activation and adaptive immune response, and to promote production of proinflammatory cytokines and chemokines in animal models. Different parasite species or strains trigger distinct IFN-I responses. For example, a Plasmodium yoelii strain can stimulate a strong IFN-I response during early infection, whereas its isogenetic strain does not. Host genetic background also greatly influences IFN-I production during malaria infections. Consequently, the effects of IFN-Is on parasitemia and disease symptoms are highly variable depending on the combination of parasite and host species or strains. Toll-like receptor (TLR) 7 , TLR9, melanoma differentiation-associated protein 5 (MDA5), and cyclic GMP-AMP synthase (cGAS) coupled with stimulator of interferon genes (STING) are the major receptors for recognizing parasite nucleic acids (RNA/DNA) to trigger IFN-I responses. IFN-I levels in vivo are tightly regulated, and various novel molecules have been identified to regulate IFN-I responses during malaria infections. Here we review the major findings and progress in ligand recognition, signaling pathways, functions, and regulation of IFN-I responses during malaria infections.

Keywords: IFN-Is, Plasmodium, immune response, signaling pathways, virulence

\section{INTRODUCTION}

Plasmodium parasites have a complex life cycle developing within a vertebrate host and a female Anopheles mosquito (Figure 1). Malaria infection begins with a mosquito bite injecting sporozoites into the skin of a vertebrate host. The sporozoites travel to the liver through the bloodstream and infect hepatocytes. In the liver, the parasites undergo multiple rounds of replication, resulting in thousands of merozoites (Prudencio et al., 2006). The merozoites are released into the blood where they rapidly infect red blood cells (RBCs) and begin intraerythrocytic cycle of replication, releasing 


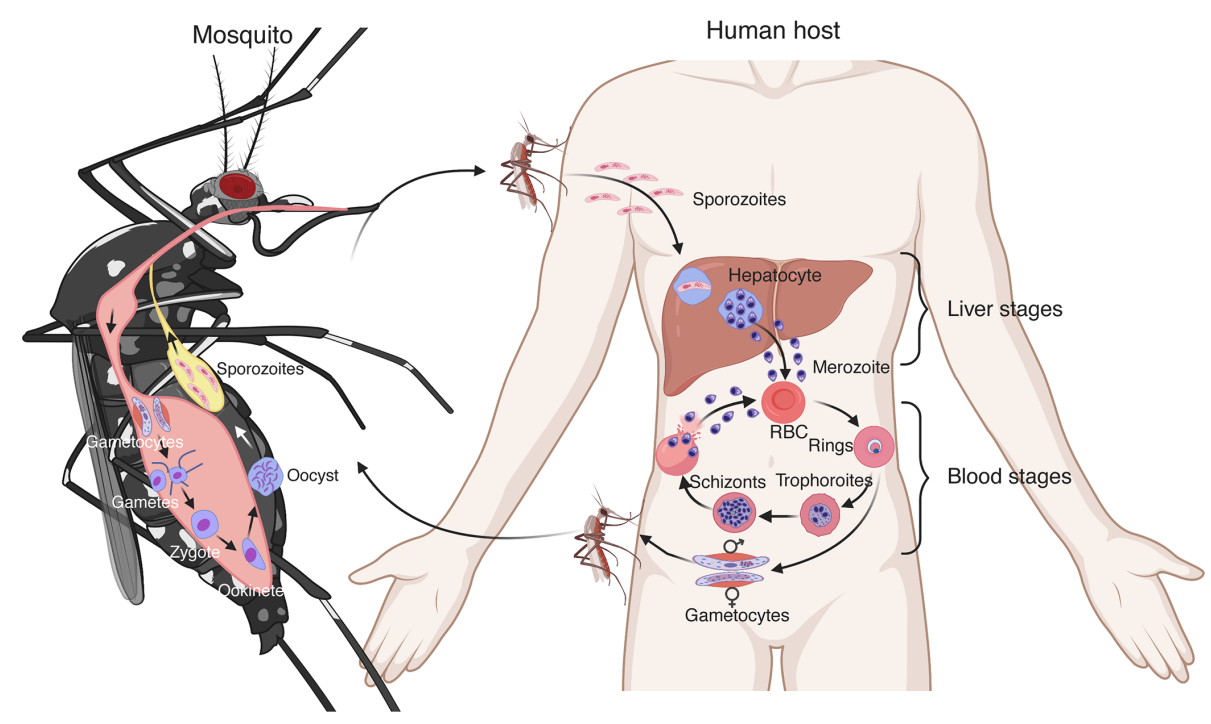

FIGURE 1 | Life cycle of a human malaria parasite. Malarial infection begins when an infected female Anopheles mosquito bites a human host and injects sporozoites into the skin. The sporozoites then travel through the bloodstream to invade hepatocytes. A sporozoite multiplies in the hepatocyte to generate thousands of merozoites. After being released from the liver, the merozoites invade red blood cells (RBCs) and develop to ring stage, trophozoite and schizont that contains 16-32 merozoites. The merozoites are released after rupture of infected RBCs to invade new erythrocytes. These blood stages of the parasite life cycle cause most malaria symptoms. A small number of merozoites develop into sexual stages, male and female gametocytes. Gametocytes are taken up by a second mosquito through a blood meal. In the mosquito midgut, the gametocytes differentiate into gametes, zygotes, ookinetes and then oocysts. A mature oocyst contains tens of thousands of sporozoites that migrate to the mosquito salivary glands to be injected into another human host to start a new cycle.

more merozoites to invade new RBCs. The erythrocytic development cycle takes approximately $24 \mathrm{~h}$ for Plasmodium knowlesi and rodent parasites such as Plasmodium yoelii, Plasmodium berghei, and Plasmodium chabaudi, $48 \mathrm{~h}$ for Plasmodium falciparum, Plasmodium vivax, and Plasmodium ovale, and $72 \mathrm{~h}$ for Plasmodium malariae. The asexual intraerythrocytic cycle is responsible for malaria symptoms (Crompton et al., 2014). During the development in RBCs, the parasites express various proteins on infected RBCs (iRBCs) and release a large amount of different materials into the bloodstream when iRBCs rupture, which trigger vigorous host immune responses and malaria signs or symptoms, including fever and chills, headache, anemia and possibly cerebral malaria.

Innate immunity is the first line of host defense against an invading pathogen. Interferons (IFNs) are produced by the host immune system and are well recognized for their role in antiviral infections. IFNs were initially described in 1957 as soluble glycoproteins with strong effects to "interfere" with the virus replication (Isaacs and Lindenmann, 1957; Isaacs et al., 1957). Today, three major groups of IFNs have been characterized: Type I IFN (IFN-I), IFN-II and IFN-III (Borden et al., 2007; Kotenko, 2011). IFN-Is consist of 13 IFN- $\alpha$ subtypes in humans (14 in mice), IFN- $\beta$, IFN- $\omega$, IFN- $\kappa$, IFN- $\epsilon$, IFN- $\zeta$, IFN- $\delta$, and IFN- $\tau$ (Pestka et al., 2004; Borden et al., 2007). Among them, IFN- $\alpha$ and IFN- $\beta$ are the most abundant and well-studied. All IFN-I subtypes signal in an autocrine and paracrine fashion through heterodimeric IFN-I receptor (IFNAR) composed of two subunits, IFNAR1 (IFN- $\alpha / \beta$ receptor $\alpha$ chain) and IFNAR2 (IFN- $\alpha / \beta$ receptor $\beta$ chain) (Uze et al., 2007; Boxx and Cheng, 2016). Binding of IFN-Is to IFNAR induces a cascade of downstream signaling events to initiate the transcription of hundreds of interferon-stimulated genes (ISGs) (Figure 2). ISGs include antimicrobial proteins, chemokines/cytokines and inflammation-inducing mediators. Many ISGs target critical molecules and pathways of a pathogen directly. Chemokines/ cytokines and their receptors enable cell-to-cell communication and cell migration, whereas negative regulators of signaling pathways maintain a balanced IFN response and cellular homeostasis. Other ISGs encode for proapoptotic proteins, leading to cell death under certain conditions (Schneider et al., 2014; Lukhele et al., 2019).

IFN-Is are some of the most common cytokines produced by the host and play important roles in both protection and pathogenesis during malaria parasite infections (Angulo and Fresno, 2002; Stevenson and Riley, 2004; Schofield and Grau, 2005; Clark et al., 2008; Dunst et al., 2017; Gotz et al., 2017). At the early stage of infection, the parasites can be recognized by the host, which induces complex immune responses, including production of IFNs and proinflammatory cytokines (Scragg et al., 1999). The pathogen components recognized by host pathogen recognition receptors (PRRs) are called pathogen-associated molecular patterns (PAMPs). PAMPs are generally evolutionarily conserved molecules, including DNA, RNA, lipopolysaccharide (LPS), peptidoglycans, glycosylphosphatidylinositol (GPI), and some 


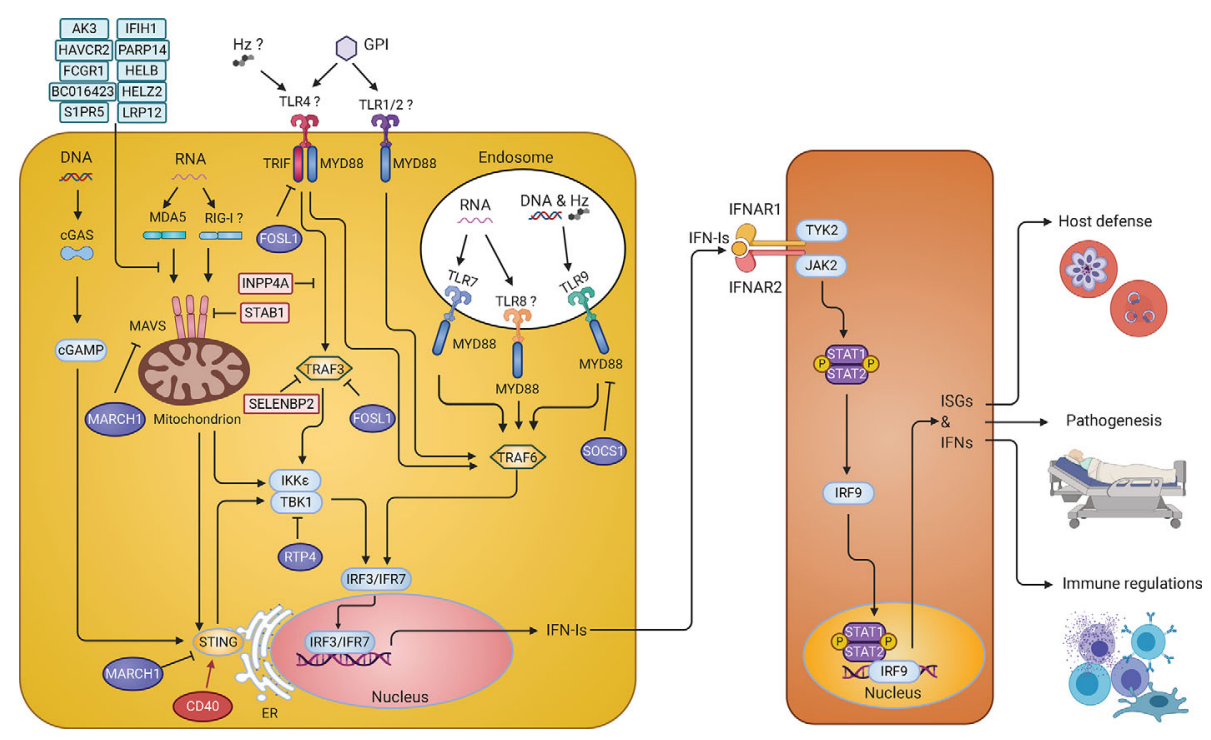

FIGURE 2 | IFN-I response and signaling pathways in malaria parasite infections. Parasite pathogen-associated molecular patterns (PAMPs) such as DNA and RNA are recognized by Toll-like receptors (TLRs) on the membrane of endosomes, including TLR7, TLR9, and possibly TLR8, and activate MYD88-TRAF6-IRF7 signaling cascade to stimulate production of IFN-Is. In the cytosol, parasite RNA is sensed by MDA5 (maybe RIG-I too) leading to the activation of MAVS-TBK1-IRF3 signaling and IFN-I production. Parasite DNA in the cytosol is sensed by cGAS, resulting in the activation of STING-TBK1-IRF3 signaling pathway to produce IFN-Is. On the cell surface, glycosylphosphatidylinositol (GPI) might be recognized by TLR4 and/or TLR1/2 that activates MYD88-TRAF6-IRF7 and TRIF-TBK1-IRF3 pathways to produce IFN-Is. Hemozoin $(\mathrm{Hz})$ can form a complex with DNA and be recognized by TLR9 or with host fibrinogen and recognized by TLR4. Hz itself can also be sensed by NLR family pyrin domain containing 3 (NLRP3). However, recognition of $\mathrm{Hz}$ by these receptors mostly leads to the production of the proinflammatory cytokines. Many molecules (in light blue or light pink) are regulators that have been shown to inhibit IFN-I responses in vitro. The roles of CD40, FOSL1, MARCH1, and RTP4 in regulating IFN-I responses were further demonstrated in vivo during malaria parasite and/or viral (RTP4) infections. These regulators either can increase (CD40 in red) or decrease (FOSL1, MARCH1, and RTP4 in purple) IFN-I production through phosphorylation and/or ubiquitination of proteins in the IFN-I response pathways. The IFN-Is secreted by the cells bind to the IFNAR to activate the STAT1/STAT2-IRF9 pathway leading to transcription of ISGs that either directly act on parasites or modulate host immune responses. Infection of Ifnar $1^{-1-}$ mice will affect downstream IFN-I signaling but may not influence (and possibly stimulate) IFN-Is production.

proteins (Beutler, 2009; Takeuchi and Akira, 2010; Kawasaki and Kawai, 2014; Wu and Chen, 2014; Brubaker et al., 2015; Vijay, 2018). During infections, some host molecules that are normally not accessible to the host immune system can be 'released' and trigger immune responses. These molecules such as high mobility box 1 (HMGB1), heat shock proteins (HSPs), SP100 protein family, and uric acid crystal are called danger-associated molecular patterns (DAMPs) (Rubartelli and Lotze, 2007; Tang et al., 2012; Kaczmarek et al., 2013; Venereau et al., 2015). The host can detect PAMPs and DAMPs by a wide range of PRRs, leading to activation of downstream pathways and production of chemokines/cytokines. PRRs are present at many different cellular locations, including the outer plasma membrane, endosomal membrane luminal surface, mitochondria outer membrane and cytosol (Kawai and Akira, 2011; Kawasaki and Kawai, 2014; Wu and Chen, 2014). Toll-like receptors (TLRs), RIG-I-like receptors (RLRs), cyclic GMP-AMP synthase (cGAS), nucleotide-binding oligomerization domain (NOD)-like receptors (NLRs), and absent in melanoma 2 (AIM2) are common receptors that recognize PAMPs and host DAMPs to initiate IFN-I and inflammatory responses.

Initially identified as potent antiviral mediators, IFN-Is have been shown to play important roles against many pathogens, including bacteria, fungi, and protozoa (Ramirez-Ortiz et al., 2011; Beiting, 2014; Boxx and Cheng, 2016; Silva-Barrios and Stager, 2017). However, IFN-Is can also cause immunopathology in acute viral infections (Davidson et al., 2014) and lead to immunosuppression during chronic viral infections (Teijaro et al., 2013; Wilson et al., 2013). Similarly, in some bacterial infections, IFN-Is have been shown to exacerbate infections and promote secondary or chronic bacterial infections (Boxx and Cheng, 2016). Dual and opposing roles of IFN-Is have also been reported in many protozoan infections (Beiting, 2014; SilvaBarrios and Stager, 2017), including conflicting roles in malaria infections. Here we review and discuss IFN-I responses during malaria infections, including parasite ligands, host receptors, IFN-I induction and regulation, and their functions in disease pathology and protection.

\section{SENSING MALARIA PARASITES: LIGANDS AND RECEPTORS}

Many PRRs have been reported to recognize different malaria PAMPs or DAMPs to initiate the immune responses (Figure 2). TLRs, RLRs and cGAS have been shown to play important roles in IFN-I responses during malaria parasite infections, whereas NLRs and AIM2 largely contribute to activation of inflammasomes (Gazzinelli et al., 2014). Malaria parasite hemozoin (Hz), RNA, DNA, GPI as well as host derived uric acid crystal, peroxiredoxins and extracellular vesicles (EVs) are examples of PAMPs and 
DAMPs that can induce IFN-I and inflammatory responses. TLRs consist of 10 members in humans (TLR1-TLR10) and 12 receptors in mice (TLR1-TLR9 and TLR11-TLR13) (Takeda et al., 2003; Nie et al., 2018). The RLRs are located at the cytoplasm and contain a DexD/H box RNA helicases domain that can detect RNA (Loo and Gale, 2011). There are three known RLRs: retinoic acid-inducible gene I (RIG-I), melanoma differentiation-associated protein 5 (MDA5), and DExH-box helicase 58 (DHX58 or LGP2). RIG-I and MDA5 are responsible for sensing double-stranded RNA (dsRNA), whereas DHX58 functions as a regulator of the RIG-I and MDA5 signaling pathways (Yoneyama et al., 2005). cGAS binds to microbial DNA and self-DNA in the cytoplasm and catalyzes the synthesis of cyclic guanosine monophosphate-adenosine monophosphate (cGAMP) (Sun et al., 2013). cGAMP acts as a second messenger to activate the downstream signal adaptor, stimulator of interferon genes (STING) (Wu et al., 2013).

TLR1, TLR2, TLR4, TLR6, TLR7 and TLR9 have been reported to recognize malaria ligands. TLR2 forms heterodimers with TLR1 or TLR6, and the heterodimers can bind to the distinct structure of parasite GPI (Campos et al., 2001; Krishnegowda et al., 2005). TLR4 can recognize parasite GPI as well as the peroxiredoxins and fibrinogen bound to $\mathrm{Hz}$ (Krishnegowda et al., 2005; Zhu et al., 2005; Barrera et al., 2011). TLR7 and TLR8 sense parasite RNA (Baccarella et al., 2013; Spaulding et al., 2016; Yu et al., 2016; Coch et al., 2019), and TLR9 recognizes parasite DNA and Hz-DNA complexes (Pichyangkul et al., 2004; Parroche et al., 2007; Wu et al., 2010; Gowda et al., 2011). The major cytosolic receptor for sensing malaria RNA is MDA5 (Liehl et al., 2014; Miller et al., 2014), although RIG-I has also been implicated to play a role in response to malaria infection (Wu et al., 2020). cGAS recognizes parasite DNA and leads to the production of IFN-Is (Yu et al., 2016; Gallego-Marin et al., 2018; Hahn et al., 2018). It is possible that different sensors are activated by parasite products at different developmental stages, leading to variation in levels of IFN-I subtypes over time. Additionally, different IFN-I subtypes can have different biological and immunological roles. For example, C57BL/6J mice infected with lymphocytic choriomeningitis virus (LCMV) $\mathrm{Cl}-13$ strain produced both IFN- $\alpha$ and IFN- $\beta$. However, IFN- $\alpha$ was found to control early viral dissemination, whereas IFN$\beta$ could suppress $\mathrm{T}$ cell responses and delay clearance of persistent virus (Ng et al., 2015; Ng et al., 2016). Additionally, IFN- $\alpha$ and IFN$\beta$ were shown to have different activities against West Nile virus infection (Sheehan et al., 2015). Whether IFN- $\alpha$ and IFN- $\beta$ play different roles in malaria parasite infections requires further investigations.

\section{DNA Sensing and Signaling Pathways}

Many studies have shown that endosomal TLR9 and cytoplasmic cGAS can recognize microbial DNA and host self-DNA in certain pathological conditions (Figure 2) (Pichyangkul et al., 2004; Beutler, 2009; Takeuchi and Akira, 2010). During malaria infections, TLR9 binds unmethylated CpG motifs of parasite DNA (Lamphier et al., 2006; Ohto and Shimizu, 2016). It is worth noting that $P$. vivax parasite that has a higher frequency of $\mathrm{CpG}$ motifs in the genome than $P$. falciparum parasite is also a stronger fever inducer (Anstey et al., 2012). Malaria parasites in the iRBCs or free merozoites are phagocytized by dendritic cells (DCs) and macrophages and enter the endosomes of these cells (Parroche et al., 2007; Wu et al., 2010; Gowda et al., 2011; Hirako et al., 2015). The endosomes then fuse to the lysosomes to form phagolysosomes and release DNA into the acidic environment. There, DNA can bind to TLR9 and activate the mitogen-activated protein kinase (MAPK)/nuclear factor kappa-light-chain-enhancer of activated $\mathrm{B}$ cells (NF- $\mathrm{KB}) /$ interferon regulatory transcription factor (IRF) 7 pathways, leading to the production of IFN-Is and proinflammatory chemokines/cytokines (Sasai et al., 2010; Kawai and Akira, 2011). For the IRF7 pathway, TLR9 recruits the toll/interleukin-1 receptor (TIR) domain containing adaptor protein (TIRAP) that binds to myeloid differentiation primary response 88 (MYD88) and interleukin-1 receptor-associated kinase 1 (IRAK1) (Kawagoe et al., 2008; Barton and Kagan, 2009; Kawai and Akira, 2010). The MYD88-IRAK1 complex further recruits tumor necrosis factor (TNF) receptor associated factor (TRAF) 3 and IRF7 that translocate into the nucleus to activate promoters of IFN-I genes (O’Neill et al., 2003; Kawai and Akira, 2008).

Parasite DNA can also enter the cytosol and activate cGAS and AIM2. cGAS synthesizes cGAMP that binds to STING (also known as MITA, ERIS or MPYS) (Ishikawa and Barber, 2008; Zhong et al., 2008; Sun et al., 2009; Jin et al., 2013) and triggers conformational changes of STING to form STING oligomers through side-by-side packing of the STING molecules (Shang et al., 2012). STING then translocates from the endoplasmic reticulum (ER) to the Golgi apparatus (Gui et al., 2019) to bind TANK binding kinase 1 (TBK1), leading to phosphorylation of interferon regulatory factor (IRF) 3 (Hornung and Latz, 2010). Phosphorylated IRF3 also translocates into the nucleus to activate IFN-I genes. DNA sensing by AIM2 generally triggers the production of inflammatory cytokines and activation of inflammasomes (Kalantari et al., 2014). Therefore, TLR9 and cGAS/STING are the major sensors for recognizing malaria parasite DNA for IFN-I responses.

\section{RNA Sensing and Signaling Pathways}

Parasite RNA from both liver and blood stages can be recognized by the host RNA sensors (Figure 2). During development in the liver, parasite RNA is mainly recognized by MDA5 (Liehl et al., 2014; Miller et al., 2014). MDA5 becomes activated after binding RNA and releases its caspase activation and recruitment domains (CARD) from the C-terminal regulatory domain (Cui et al., 2008). Activated MDA5 recruits mitochondrial antiviral-signaling protein (MAVS) through homotypic CARD-CARD domain interactions (Kawai et al., 2005; Meylan et al., 2005; Seth et al., 2005; Xu et al., 2005). MAVS forms prion-like structures and further recruit TRAF3, TBK1 and IאB kinase epsilon (IKKe) to phosphorylate IRF3 and IRF7, leading to IFN-I production (Hou et al., 2011; Jacobs and Coyne, 2013). MAVS can also bind to TRAF6 and activate NF- $\mathrm{KB} / \mathrm{IKK}$ complex, resulting in the production of inflammatory cytokines (Belgnaoui et al., 2011). TLR7 and MDA5 can also sense parasite RNA from blood stages (Baccarella et al., 2013; Wu et al., 2014; Spaulding et al., 2016; Yu et al., 2016). Similar to TRL9, TLR7 activates the MYD88 and IRF7 pathway to produce IFN-Is (Baccarella et al., 2013; Yu et al., 2016). TLR7 and MDA5 are the major sensors for malaria parasite RNA for IFN-I responses.

Additionally, RNA polymerase III (pol III) was reported to convert AT rich viral DNA into dsRNA that can be recognized 
by the RLRs (Chiu et al., 2009). Pol III deficiency led to reduced IFN-I production in Raw 264.7, but not in HEK293T cells (Sharma et al., 2011; Wu et al., 2014). This discrepancy is likely due to difference in gene expression between the two cell lines including the lack of TLR expression in the HEK293T cells (Hornung et al., 2002). Interestingly, RIG-I expression was detected in a study using trans-species expression quantitative trait locus (ts-eQTL) analysis after infection of 24 parasite progeny from a $P . y$. yoelii $17 \mathrm{XNL} \times P . y$. nigeriensis $\mathrm{N} 67$ cross (Wu et al., 2020). Additionally, messenger RNA (mRNA) and protein levels of IFN- $\beta$ could be significantly suppressed by RNAi knockdown of the genes encoding MDA5, RIG-I, or RNA pol III after P. yoelii DNA stimulation (Wu et al., 2014). However, whether RIG-I and Pol III play a role in the recognition of parasite DNA or RNA in vivo requires further investigations.

\section{Responses to GPI}

GPI exists in many eukaryote species and is attached to a protein during posttranslational modification (Brown and Waneck, 1992). The major function of GPI is binding to certain functional proteins at the cell surfaces (Ferguson et al., 1999). GPI contains a conserved ethanolamine phosphate-substituted oligosaccharide moiety that links to phosphatidylinositol with an alpha glycosidic bond (Ropert and Gazzinelli, 2000). The structures of GPIs in different species vary, mainly in the lipid substituents, sugar chain length, or ethanolamine phosphate residues attached to the conserved glycan moiety. Malaria GPI is one of the earliest identified parasite PAMPs (Schofield and Hackett, 1993). The detailed structures of parasite GPI have been studied using biochemical analysis and mass spectrometry (Naik et al., 2000). The structures of parasite GPIs are different from human GPIs and can be recognized by the host's immune system. Studies have shown that malaria GPI is sensed by TLRs on the surface of macrophages or DCs, mainly through the TLR1-TLR2 heterodimers and TLR4 (Krishnegowda et al., 2005; Zhu et al., 2005; Lu et al., 2006; Zhu et al., 2009). Similar to TLR7 and TLR9, TLR1-TLR2 and TLR4 recruit MYD88 to activate the NF- $\kappa \mathrm{B}$ and IRF7 pathways, leading to the production of IFN-Is and inflammatory cytokines. TLR 4 can also recruit a protein called TIR-domain-containing adapter-inducing interferon- $\beta$ (TRIF) that further forms a complex with IKKe and TBK1 to phosphorylate and activate IRF3 and IRF7 (Sweeney et al., 2004; Kawagoe et al., 2008; Beutler, 2009; Ning et al., 2011; Kawasaki and Kawai, 2014). However, the majority of studies on malaria GPI have focused on activation of inflammatory cytokines. One study shows that GPI can induce interferon-sensitive response element (ISRE) activation in vitro, which suggests that GPI may also stimulate IFN-I production via TLR4 (Yao et al., 2016). Since inflammatory responses by malaria infection can inhibit MYD88-IRF7dependent IFN-I signaling (Yu et al., 2018), it is possible that the strong pro-inflammatory responses stimulated by parasite GPI can influence other IFN-I pathways indirectly.

\section{Parasite $\mathrm{Hz}$ and Host Responses}

$\mathrm{Hz}$ is a brown pigment that is formed during the digestion of hemoglobin in the digestive vacuole (DV) of blood stage parasites (Pandey and Tekwani, 1996; Arese and Schwarzer, 1997; Pagola et al., 2000; Jani et al., 2008; Olivier et al., 2014).
$\mathrm{Hz}$ is captured by immune cells after rupture of iRBCs (Celada et al., 1983; Ho and Webster, 1989; Arese and Schwarzer, 1997). There is no known receptor for $\mathrm{Hz}$; however, it can trigger immune responses in multiple ways. $\mathrm{Hz}$ carrying parasite DNA is a potent activator of TLR9, leading to production of ISGs and pro-inflammatory cytokines (Parroche et al., 2007). The parasite DNA does not colocalize with $\mathrm{Hz}$ that is synthesized in the DV (Jaramillo et al., 2009). Therefore, $\mathrm{Hz}$ probably binds DNA released from dead cells or $\mathrm{DV} . \mathrm{Hz}$ can also form a complex with the host fibrinogen that is then recognized by TLR4 or CD11b/CD18-integrin on monocytes, leading to production TNF and reactive oxygen species (ROS) (Barrera et al., 2011).

$\mathrm{Hz}$ may cause immune cell dysfunction or apoptosis after phagocytosis by the monocytes or macrophages. Studies have shown that macrophages became functionally impaired after ingestion of $P$. falciparum-infected erythrocytes or isolated $\mathrm{Hz}$ due to a long-lasting oxidative burst (Schwarzer et al., 1992). Monocytes or macrophages ingesting a large amount of iRBC or $\mathrm{Hz}$ may undergo apoptotic death with low levels of cytokine production due to phagolysosomal acidification (Schwarzer et al., 2001; Wu et al., 2015b). Hz can also inhibit differentiation and maturation of human DC by impairing expression of major histocompatibility complex class II antigen (Schwarzer et al., 1998; Skorokhod et al., 2004). Hz induces phagolysosomal destabilization leading to release of lysosomal contents to the cytosol, including DNA or RNA to induce IFN-I responses. Additionally, $\mathrm{Hz}$ itself can activate the NLRP3 inflammasome through Lyn and Syk signaling pathways (Dostert et al., 2009; Olivier et al., 2014). An unidentified ligand released from the parasite lysosome (or DV) has been reported to activate NLRP12 inflammasome (Ataide et al., 2014). Overall, $\mathrm{Hz}$ is considered as a danger signal which can activate diverse pathways to influence IFN-I production and inflammatory responses (Dostert et al., 2009; Shio et al., 2009).

\section{Extracellular Vesicles (EVs) and Host Responses}

EVs are lipid bilayer-delimited particles derived from iRBCs or other host cells (Babatunde et al., 2020). Based on their size and biological functions, EVs are classified into two forms: exosomes and microvesicles. Exosomes are smaller vesicles ranging from 30 to $150 \mathrm{~nm}$ in diameter and are usually released by reticulocytes during differentiation and maturation (Johnstone et al., 1987). Microvesicles are larger vesicles with sizes of $150 \mathrm{~nm}$ to $1-2 \mu \mathrm{m}$ in diameter and are produced by the plasma membrane budding and fission (Tricarico et al., 2017). EVs contain lipids, proteins, and nucleic acids (DNA and RNA) (Laulagnier et al., 2004; Nolte-'t Hoen et al., 2012; Tauro et al., 2012; Ridder et al., 2014; Sinha et al., 2014; Williams et al., 2014; Babatunde et al., 2018). Malaria parasite infection causes a strong inflammatory response, and inflammatory cytokines such as TNF- $\alpha$ can greatly contribute to the production of the EVs by almost all cell types (Combes et al., 1999; Freyssinet, 2003). Studies have shown that EVs containing parasite materials are potential triggers of proinflammatory innate immune responses (Couper et al., 2010). In animal models, blockage of EV production in mice resulted in complete resistance to cerebral malaria development (Combes et al., 2005; Penet et al., 2008). Transfer of 
EVs from P. berghei ANKA-infected mice caused damages to the blood-brain barrier in the recipient mice (El-Assaad et al., 2014). Parasite DNA in the EVs can be detected by cGAS to activate the STING-dependent pathway leading to the production of IFN-Is and inflammatory cytokines (Sisquella et al., 2017). Parasite RNA in the EVs, on the other hand, will activate MDA5 and trigger the MAVSdependent pathway to produce IFN-Is (Ye et al., 2018). EVs also contain microRNA (miRNA) and mRNA. mRNA can be translated into protein in the recipient cells; meanwhile, miRNA may regulate gene expression in the target cells (Ratajczak et al., 2006; Valadi et al., 2007). Similar to Hz, EVs and their contents can influence several host pathways, including IFN-I responses.

\section{HOST CELLS PRODUCING IFN-IS}

IFN-Is can be produced by several types of host cells during malaria infections. Plasmodium parasites reside in different cell types during their life cycle. In the liver, the parasites develop mostly in the hepatocytes, and large amount of parasite materials are synthesized and released within the hepatocytes. MDA5 in hepatocytes can recognize parasite RNA from liver stages leading to IFN-I production (Liehl et al., 2014; Miller et al., 2014). In the blood stage infections, IFN-I production is more complicated. The parasites reside in RBCs that lack PRRs and the machinery for IFN-I responses. Peripheral blood mononuclear cell (PBMC) cultures stimulated with iRBC can produce IFN- $\alpha$ in vitro (Montes de Oca et al., 2016). Other studies find that plasmacytoid dendritic cells (pDC) are the major source of IFN-Is (deWalick et al., 2007; Voisine et al., 2010). Splenic conventional dendritic cells (cDC) are an alternative source of IFN-Is (Voisine et al., 2010; Haque et al., 2014). Macrophages can also produce IFN-Is during blood stage infections (Spaulding et al., 2016; Yu et al., 2016). NK cells from healthy donors' PBMCs co-cultured with $P$. falciparum 3 D7-infected erythrocytes upreguated IFN- $\alpha$ related genes (Grangeiro de Carvalho et al., 2011). Therefore, hepatocytes and DCs are the main sources of IFN-Is during liver and blood stage infections, although many other cell types may also contribute to the IFN-I production.

\section{IFN-I RESPONSES TO LIVER STAGES}

Although infection at the liver stages is generally asymptomatic, studies have shown that a complex series of immune events occur during liver stage development, including IFN-I responses (Liehl and Mota, 2012; Liehl et al., 2014; Miller et al., 2014). Transcriptomic analysis of liver stages showed an IFNAR1dependent IFN-I response in hepatocytes, which was not restricted to Plasmodium species ( $P$. berghei and $P$. yoelii) nor mouse host strains (BALB/c and C57BL/6) (Liehl et al., 2014). Plasmodium parasite RNA was reported to be recognized by MDA5 in hepatocytes (and possibly other receptors). Furthermore, the magnitude of IFN-I responses was parasite dose and replication dependent. Leukocytes were mobilized as effectors by IFN-Is to eliminate the parasites (Liehl et al., 2014) (Figure 3A). IFN-I signaling helps recruit NK cells and NKT cells, especially IFN- $\gamma$ secreting CD1d-restricted NKT cells to eliminate the liver stage parasites (Miller et al., 2014). The IFN-I responses also benefit the host from an immediate liver stage reinfection by significantly reducing the liver parasite load as well as blood stage parasitemia (Liehl et al., 2015). Similarly, attenuated P. yoelii parasites that only develop to late liver-stage forms induce significant IFN responses (including IFN-Is and IFN- $\gamma$ ) and provide cross protection against a secondary liver stage infection ( $P$. yoelii or $P$. berghei) (Miller et al., 2014). A distinct population of CD11 ${ }^{+}$cells was also observed to invade the liver at $36-40 \mathrm{~h}$ post $P$. y. yoelii $17 \mathrm{XNL}$ sporozoite inoculation (Kurup et al., 2019a). These monocyte derived CD11c ${ }^{+}$ dendritic cells (DCs) showed strong IFN-I profiles, with highly upregulated expression of key molecules such as TLR7 and IRF7 in the IFN-I pathways. The CD11c $\mathrm{c}^{+}$DCs acquired and presented parasite antigen by phagocytosing infected hepatocytes, to prime $\mathrm{CD}^{+} \mathrm{T}$ cells, the major mediators in liver stage protection (Kurup et al., 2019a). P. berghei radiation-attenuated sporozoites (RAS) could also induce liver $\mathrm{CD} 8 \alpha^{+}$DCs that activated $\mathrm{CD} 8^{+} \mathrm{T}$ cells in the liver (Jobe et al., 2009), although the activation level was much weaker than that induced by normal sporozoites (Parmar et al., 2018). The accumulation and activation of CD8 $\alpha^{+}$DCs was associated with higher-levels s of CCL-20, CCL-21, and IFN-Is (Parmar et al., 2018). Interestingly, a baculovirus-induced innate immunity also provided complete protection against subsequent $P$. berghei sporozoite infection or existing liver stage infection, and neutralization of IFN- $\alpha$ could abolish this effect (Emran et al., 2018). These studies provide mechanistic supports for some earlier observations that interferon inducers have better protection against $P$. berghei sporozoite-induced infection than that by blood stage infection (Jahiel et al., 1968a; Jahiel et al., 1968b; Jahiel et al., 1970). The prevalent pathways of IFN-I induction are through activation of cytosolic receptors that recognize parasite nucleic acids. A significant amount of parasite RNA was detected in nonhepatocytes during liver stage development (Kurup et al., 2019a), suggesting that DCs and other cells may also be sources of IFN-Is. Together, these studies show that during acute liver stage infection, parasite nucleic acids from the rapidly replicating sporozoites can trigger IFN-I responses and production. The IFN-Is then lead to an influx of leukocytes, including NK, NKT and DCs, to the liver and prime the adaptive immunity, especially $\mathrm{CD}^{+} \mathrm{T}$ cells, to subsequently eliminate the parasites. Until now, only parasite RNA has been documented to induce IFN-Is in the hepatocytes. In addition, it has been reported that many sporozoites inoculated into the skin of a mouse migrated to the lymph-nodes and were phagocytosed by CD8 $\alpha^{+}$DCs to induce $\mathrm{CD}^{+} \mathrm{T}$ cell responses (Radtke et al., 2015), but whether this process also triggers IFN-I responses requires further investigations.

Sterile protection against Plasmodium infection can be achieved through exposure to radiation- or genetically-attenuated sporozoites that are able to infect but cannot replicate within the hepatocyte, or through exposure to sporozoites under chloroquine chemoprophylaxis (Mo and McGugan, 2018). It appears that IFNIs are part of protective immunity during normal or attenuated sporozoite infection; however, the roles of IFN-Is in infections by sporozoites attenuated by various methods has not been well 

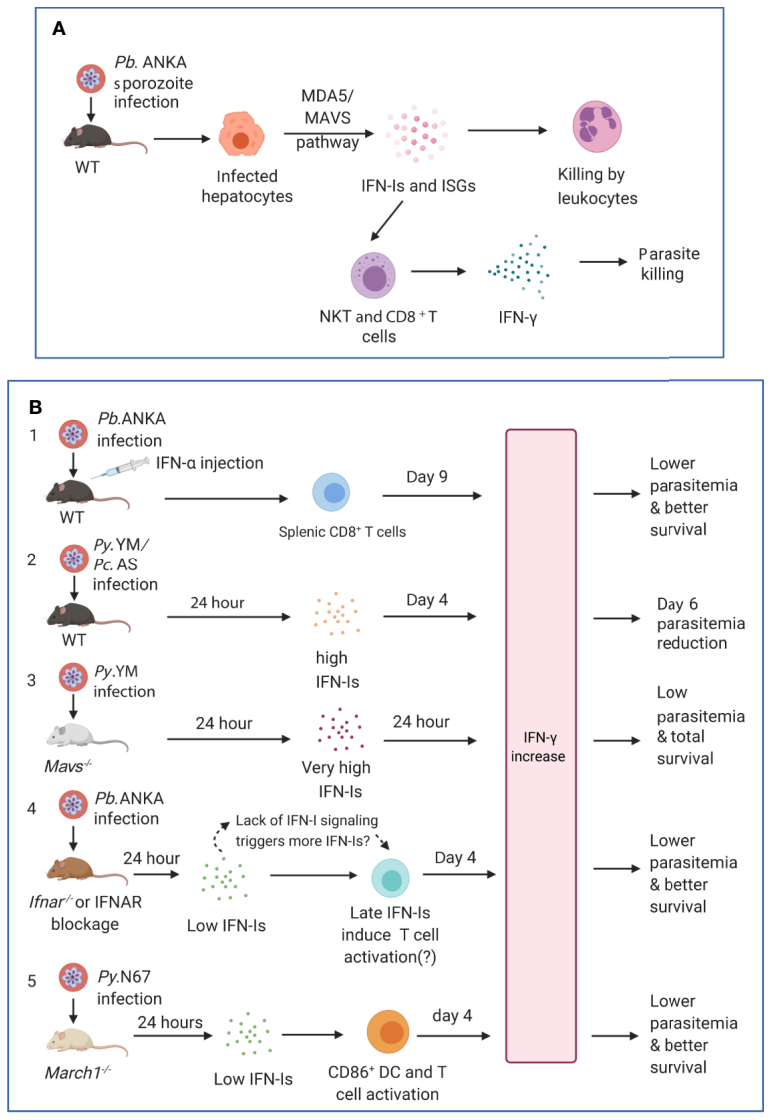

FIGURE 3 | Timing and levels of interferon production may determine the outcomes of malaria parasite infections. (A) The protection against liver stages relies on the activation of innate immunity involving IFN-I responses and immune cell infiltration. Infection of C57BL/6 mice with P. berghei ANKA liver-stages induces an IFN-I response through MDA5 signaling pathway in hepatocytes. Infiltrating leukocytes (macrophages and neutrophils) are mobilized to the vicinity of infected hepatocytes by IFN-I signaling (Liehl et al., 2014). IFN- $\gamma$-secreting immune cells, in particular CD1d-restricted NKT cells, are also likely the main players responsible for the innate elimination during liver stage (Miller et al., 2014). (B) Several models show that IFN-Is likely function through regulating IFN- $\gamma$ production, T cell activation, and adaptive immune response to influence parasitemia and disease severity during blood stage infections. First, several studies showed that injections of IFN- $\alpha$ and/or IFN- $\beta$ were protective (Supplementary Table 1). C57BL/6 mice infected with $P$. berghei ANKA and injected with IFN- $\alpha$ had significantly $(P<0.05)$ higher levels of IFN- $\gamma(772 \pm 73 \mathrm{pg} / \mathrm{ml}$, day 9 pi) than those receiving diluent $(180 \pm 14 \mathrm{pg} / \mathrm{ml})$, reduced parasitemia, and better host survival (Vigario et al., 2007). Second, C57BL/6 mice infected with P. y. nigeriensis N67 or P. chabaudi AS had elevated levels of IFN- $\alpha(\sim 320$ and $\sim 450$ pg/ml, respectively) 24 h pi (Kim et al., 2012 ; Wu et al., 2020). Mavs $^{-/-}$mice infected with P. y. nigeriensis N67 or Ifnar ${ }^{-1-}$ mice infected $P$. chabaudi AS had increased day 6 parasitemia (parasitemia increases in Ifnar ${ }^{-1-}$ mice may not be significant but had reduced ability to resolve parasitemia later) (Noisine et al., 2010; Kim et al., 2012; Wu et al., 2014). IFN-Is appear to work with IFN- $\gamma$ in controlling parasitemia during early infection. Compared with $P$. y. yoelii YM infected WT C57BL/6 mice that produced very low IFN-Is $24 \mathrm{~h}$ pi, $P$. $y$. nigeriensis N67-infected mice had significantly higher IFN- $\alpha / \beta$, IFN- $\gamma$ and IL-6 $24 \mathrm{~h}$ pi (Wu et al., 2020). Additionally, Ifnar $1^{-/-}$Ifngr $1^{-/-}$mice infected with $P$. chabaudi AS exhibited higher mortality than WT or Ifnar $1^{-1-}$ mice and were not able to completely clear parasites (Kim et al., 2012). Third, Mavs ${ }^{-/-}$mice infected with $P$. $y$. yoelii YM produced very high levels of IFN- $\alpha(\sim 2,800 \mathrm{pg} / \mathrm{ml})$, IFN- $\beta(\sim 2,000 \mathrm{pg} / \mathrm{ml})$ and IFN- $\gamma(>1,200 \mathrm{pg} / \mathrm{ml}) 24 \mathrm{~h}$ pi and all survived the infections (Yu et al., 2016). All these models suggested early production ( $24 \mathrm{~h}$ ) of IFN- $\alpha / \beta$ and IFN- $\gamma$ can help control parasitemia and may improve host survival. Fourth, in $P$. berghei ANKAinfected WT C57BL/6 mice, low levels of IFN- $\alpha / \beta$ were observed $24 \mathrm{~h}$ after pi (Haque et al., 2011; Haque et al., 2014). Depletion or blockage of IFN-I signaling using Ifnar ${ }^{-1-}$ mice or anti-IFNAR antibody treatment results in higher levels of IFN- $\gamma$, better parasite control, and improved host survival (Haque et al., 2011; Haque et al., 2014). Interestingly, higher levels of IFN- $\alpha$ were observed in WT mice $48 \mathrm{~h} \mathrm{pi}(\sim 70 \mathrm{pg} / \mathrm{ml} \mathrm{IFN}-\alpha$ vs $\sim 20 \mathrm{pg} / \mathrm{ml}$ at $24 \mathrm{~h})$ and day $4 \mathrm{pi}(\sim 200 \mathrm{mg} / \mathrm{ml}$ IFN- $\alpha)$, suggesting that blockage of IFN-I signaling may stimulate IFN-I response. Unfortunately, the IFN-I levels in Ifnar ${ }^{-1-}$ mice or mice treated with anti-IFNAR were not measured at additional time points. Similarly, anti-IFNAR antibody treatment of C57BL/6 mice infected with P. y. yoelii 17XNL significantly reduced days 16 and 21 parasitemia through inhibition of T regulatory 1 response, enhancement of Tfh cell accumulation and better humoral immunity (Zander et al., 2016). Again, the levels of IFN-Is in the anti-IFNAR treated animals were not reported. Serum levels of IFN- $\gamma$ between anti-IFNAR antibody treated and non-treated mice were similar at day 16 pi when parasitemia began to show significant difference. It is possible that the lack of IFN-I signaling in these models prompts the system to produce more IFN-Is, and that IFN-Is and IFN- $\gamma$ work together through regulating immune cell populations and antibody production to control the infections. Fifth, March $1^{-1-}$ mice infected with $P$. $y$. nigeriensis N67 (or P. y. yoelii YM) had low levels of IFN-ls $24 \mathrm{~h}$ pi but had significantly increased IFN- $\gamma$ and IL-10 day 4 pi due to decreased degradation of CD86/ MHCII and T cell activation, leading to reduced parasitemia and better host survival (Wu et al., 2020). IFN- $\gamma$ was shown to be a key player in controlling parasitemia and host survival. These observations suggest key roles of early IFN-Is (24 h pi) and IFN- $\gamma$ in later stages of infection (day 4 or later) and emphasize the importance of measuring IFN-Is, IFN- $\gamma$, and other cytokines during the course of blood infection for better understanding of protection mechanisms mediated by IFNs. Pb.ANKA, $P$. berghei ANKA; Py.N67, P. y. nigeriensis N67; Py.YM, P. y. yoelii YM; PC.AS, P. chabaudi AS. 
studied. Late liver stage-arresting and replication-competent (LARC) of genetically-attenuated sporozoites have been shown to provide good cross-stage and cross-species protection in mice (Butler et al., 2011; Vaughan et al., 2018). However, a recent study using $P$. yoelii LARC sporozoites showed that the liver stageengendered IFN-I signaling impaired hepatic $\mathrm{CD}^{+} \mathrm{T}$ cell responses, which is critical for liver stage protection. Compared with wildtype (WT) mice, Ifnar $1^{-/-}$mice exhibited superior protection due to greater $\mathrm{CD}^{+} \mathrm{T}$ cell memory and superior $\mathrm{CD}^{+} \mathrm{T}$ effector function (Minkah et al., 2019). However, the protective immune mechanisms in the Ifnar $1^{-1-}$ mice after infections with WT and LARC sporozoites can be quite different, and the observations in IFN-1 signaling-deficient mice infected with defective sporozoites may not represent the true protective immunity in normal malaria infections.

\section{IFN-IS IN BLOOD STAGE INFECTIONS}

Confounding roles of IFN-Is have been reported during bacterial and viral infections (Carrero, 2013). IFN-Is can be protective against bacterial and viral infections by promoting the induction of TNF- $\alpha$, nitric oxide, and other cytokines. In contrast, IFN-I signaling can also induce suppression of adaptive immune responses during chronic infection with LCMV and acute infections with intracellular bacteria (Carrero, 2013). During malaria blood stage infections, both positive and negative effects of IFN-Is on protection have also been reported in different rodent malaria models and in human infections, which may mirror the observations in acute and chronic infections of bacteria and viruses.

\section{Malaria Blood Stage Infection Stimulates IFN-Is Responses}

A single sporozoite can generate thousands of merozoites that are released to the bloodstream to invade RBCs. In 1968, almost 10 years after the discovery of IFNs, IFNs were reported to be present in the serum of $P$. berghei-infected mice (Huang et al., 1968). However, in a later study, no IFN activity was detected in adult human sera during malaria infection (majority $P$. vivax infection) using virus titration assay (Rytel et al., 1973). Another early study found that acute $P$. falciparum infection induced strong IFN-I responses, especially IFN- $\alpha$, in children, which was correlated with both parasitemia and NK cell activity (Ojo-Amaize et al., 1981). The differences in these studies could be due to variation in parasite species, infection stage, the time of measurement, as well as host age. Lack of sensitive methods to directly measure IFN-Is at the time of the studies could also be another reason. Later, human P. falciparum schizonts and parasite lysate were shown to activate human pDC and murine DCs to produce IFN-Is through the TLR-9 dependent pathway, and elevated serum levels of IFN-Is were detected in $P$. falciparum patients (Pichyangkul et al., 2004). Similarly, P. berghei blood stage infection led to IFN-I production by both mouse $\mathrm{pDCs}$ and cDCs (deWalick et al., 2007). Blood stage P. yoelii infection induced IFN-I responses by CDC via the MDA5 pathway (Wu et al., 2014). In a transcriptomic study of infections with multiple $P$. yoelii strains, TLR3/7, TLR9, cGAS, MDA5, and RIG-I were shown to be the upstream regulators significantly activated on day 1 after $P$. $y$. nigeriensis N67 infection (Xia et al., 2018). pDCs were primed by activated $\mathrm{CD} 169^{+}$macrophages upon STING-mediated sensing of parasites in the bone marrow to generate a robust IFN-I responses in P. y. yoelii YM blood stage infection (Spaulding et al., 2016). In controlled human malaria infection (CHMI), IFN-Is could be produced throughout the course of infection (Montes de Oca et al., 2016). However, induction of IFN-I production is parasite strain dependent; for example, $P$. yoelii nigeriensis N67 can stimulate an early peak of IFN-Is, whereas mice infected with $P . y$. nigeriensis N67C or P. y. yoelii YM produce low levels of IFN-Is (Wu et al., 2014; Wu et al., 2015a; Wu et al., 2020). The parasitemia in mice infected with $P$. y. nigeriensis N67, P. y. nigeriensis N67C and $P$. y. yoelii $\mathrm{YM}$ strains are similar at $24 \mathrm{~h}$ pi (Pattaradilokrat et al., 2014), suggesting that the differences in IFN-I levels are unlikely due to variation in parasitemia. A C741Y amino acid substitution in the protein trafficking domain of the P. y. nigeriensis N67 erythrocyte binding-like (PyEBL) protein was shown to affect host immune response, including IFN-I pathways, T cell activation and IgG switching by enhancing phosphatidylserine (PS) exposure on iRBC surface and phagocytosis (Peng et al., 2020). Other genes including those in a locus of parasite chromosome 13 were also significantly linked to expression of many ISGs (Wu et al., 2015a).

\section{Protective IFN-I Responses Against Parasitemia and Host Mortality}

IFN-Is have been shown to be protective against blood stage malaria parasites including suppression of parasitemia and/or improved host survival (Figure 3B). Upon activation of IFN-I signaling, ISGs including chemokines/cytokines and other inflammation-inducing mediators are produced to eliminate parasites residing in the host RBCs. Mice deficient of MDA5 or MAVS had compromised ability to control parasitemia day 6 after injection of $P . y$. nigeriensis N67 iRBCs (Wu et al., 2014). A strong IFN-I transcriptional signature was also found in circulating neutrophils from $P$. vivax-infected patients and in P. chabaudi-infected mice (Rocha et al., 2015). IFN-I signaling recruited neutrophils that contributed to parasite control but also caused liver damage (Rocha et al., 2015). By blocking STING- and/or MAVS-mediated IFN-I signaling, strong early TLR7-mediated IFN-I responses during P. y. yoelii YM blood stage infection helped the host control the parasitemia as well as lower mortality (Yu et al., 2016) (Figure 3B). In addition, increased transcription of ISGs in pDCs was reported after P. chabaudi blood stage infection or in vitro iRBC stimulation, although minimal effects of pDCs or IFN-I signaling on P. chabaudi AS infection were observed (Voisine et al., 2010). The dynamics of parasitemia and host survival are different between mice infected with $P$. $y$. nigeriensis $\mathrm{N} 67$ and $P$. chabaudi AS, but these infections shared elevated levels of IFN-Is $24 \mathrm{~h}$ pi (300-500 pg/ml) (Kim et al., 2012; Wu et al., 2020) and reduced days 6 and 7 parasitemia (the differences in parasitemia for some $P$. chabaudi AS infections may not be statistically significant) (Voisine et al., 2010; Kim et al., 2012; Wu et al., 2020). It is important to note that different mouse strains were used in the studies (129Sv/Ev, C57BL/6, BALB/c for $P$. chabaudi and C57BL/6 for $P$. yoelii infections). In addition, TLR7 signaling was the major pathway for IFN-I response in 
P. chabaudi AS infection, whereas MDA5 was the major RNA sensor in P. y. nigeriensis N67 infections (Baccarella et al., 2013; Wu et al., 2014). The differences in parasitemia control and host survival in mice infected with different strains of $P$. chabaudi and $P$. yoelii could also be partly due to variations in immune mechanisms controlling these infections. In P. chabaudi infection, $\mathrm{CD} 4^{+} \mathrm{T}$ cells appear to play a major role in protective immunity, although $\mathrm{B}$ cells and antibodies can also contribute to the protection, including B cell regulation of Th cell responses during primary infection (Langhorne et al., 1990; von der Weid et al., 1994; Xu et al., 2000). For some P. yoelii infections, the defense mechanism is mostly mediated by humoral factors in the absence of demonstrable cellmediated immunity (Murphy and Lefford, 1979; Freeman and Parish, 1981). IFN-Is have also been shown to enhance humoral immunity and promote isotype switching by stimulating dendritic cells in vivo (Le Bon et al., 2001). A substitution of C741Y in the P. $y$. nigeriensis $\mathrm{N} 67$ was indeed linked to increased levels of IFN- $\alpha / \beta, \mathrm{T}$ helper cell differentiation, and antibody isotype switching (Peng et al., 2020). Stimulation of an early IFN-I response may influence the direction of host immune responses such as $\mathrm{CD} 4^{+} \mathrm{T}$ cell activation and later antibody production. Whether and how IFNIs regulate host immune mechanisms, including $\mathrm{T}$ cell activation and antibody production require additional investigation.

Furthermore, continuous injection of purified recombinant human IFN- $\alpha$ suppressed blood stage parasitemia for two P. yoelii strains (265 BY and 17XNL) by inhibiting the production of reticulocytes, which were preferentially invaded by these parasite strains, but not for the strain of $P$. vinckei petteri that infects only mature red blood cells (Vigario et al., 2001). Constant human recombinant IFN- $\alpha$ treatment also resulted in enhanced survival, which was associated with an IFN- $\gamma$-mediated decrease of parasitemia, expression of intracellular adhesion molecule-1 (ICAM-1 or CD54) in the brain, and $\mathrm{CD}^{+} \mathrm{T}$ cells sequestration (Vigario et al., 2007) (Figure 3B). ICAM-1 is a cell surface glycoprotein that is typically expressed on endothelial and immune cells; it plays an important role in cell-cell adhesion, extravasation, signal transduction, and inflammation (Bui et al., 2020). Increased expression of ICAM-I in the brain microvasculature has been implicated in the development of cerebral malaria (CM). Absence of ICAM-1 correlates with a decrease of macrophage and iRBC sequestration in the brain and lung capillaries leading to a less severe thrombocytopenia and reduced mortality (Favre et al., 1999). In addition, administration of murine IFN- $\beta$ after $P$. berghei ANKA infection increased host survival and improved blood-brain barrier function without altering systemic parasitemia. Injection of IFN- $\beta$ also downregulated CXCL9 and ICAM-1 expression in the brain, reduced serum TNF- $\alpha$ level, and decreased T-cell infiltration in the brain (Morrell et al., 2011). The observations of protection in different parasite models are quite diverse. It is too early to draw a general conclusion on protective mechanisms. More studies on protection mechanisms are required.

\section{IFN-I Suppression of DC and T Cell Activation}

DCs play a central role as a bridge between innate and acquired immunity (Wykes and Good, 2008). IFN-Is were shown to modulate DC function and impair the development of protective
IFN- $\gamma$ producing $\mathrm{CD} 4^{+}{ }^{-}$- bet $^{+} \mathrm{T}$ cells for parasite control during $P$. berghei ANKA and $P$. chabaudi AS infections (Haque et al., 2011; Haque et al., 2014). CD4 ${ }^{+}$T cells have been shown to produce IFN- $\gamma$ and macrophage colony-stimulating factor (M-CSF or CSF1) that are important for the activation and expansion of $\mathrm{CD} 169^{+}$ macrophages to control malaria blood-stage infection (Fontana et al., 2016; Kurup et al., 2019b). IFN-I signaling impaired CD8 ${ }^{-}$ cDC function to prime IFN- $\gamma$-producing T helper type 1 (Th1) cells for parasite control (Haque et al., 2014), and IFN-I signaling deficiency promoted $\mathrm{CD} 4^{+} \mathrm{T}$-cell-dependent parasite control thus reducing the onset of severe clinical symptoms and fatal cerebral pathology (Haque et al., 2011) (Figure 3B). IFN-Is and IFN- $\gamma$ were involved in activation-induced $\mathrm{cDC}$ death during $P$. berghei ANKA infection (Tamura et al., 2015). In a non-lethal P. y. yoelii 17XNL blood stage infection, IFN-Is directly induced T-bet and Blimp-1 expression to promote $\mathrm{T}$ regulatory 1 ( $\mathrm{Tr} 1$ ) cell responses (Zander et al., 2016). The Tr1 cells then secreted interleukin 10 (IL-10) and IFN- $\gamma$ to restrict $T$ follicular helper (Tfh) cell accumulation and limit parasite-specific antibody responses. Furthermore, IFN-Is were shown to suppress innate immune cell function and IFN- $\gamma$ production by parasite-specific $\mathrm{CD} 4^{+} \mathrm{T}$ cells as well as to promote the development of IL-10-producing Tr1 cells (Montes de Oca et al., 2016). On the other hand, IFNAR1-deficiency accelerated humoral immune response and parasite control by boosting the inducible $\mathrm{T}$ cell co-stimulator (ICOS) signaling (Sebina et al., 2016). IFNAR1signalling also impaired germinal center B-cell formation, Ig-class switching, and Tfh cell differentiation thus impeding the resolution of non-lethal blood-stage infection of $P . y$. yoelii 17XNL and $P$. chabaudi AS.

\section{IFN-Is Promote Inflammation and Host Death}

In other studies, IFN-I signaling appears to promote production of inflammatory cytokines/chemokines and pathogenesis of experimental cerebral malaria (ECM). Key mediators of ECM, including $P$. berghei ANKA-induced brain sequestration of $\mathrm{CXCR}^{+}{ }^{+}$-activated $\mathrm{CD} 8^{+}$T cells, granzyme B, IFN- $\gamma$, CXCL9, and CXCL10, were attenuated in IFN-I signaling deficient mice (Palomo et al., 2013). Microglia from mice infected with P. berghei ANKA showed signature of IFN-I signaling that was responsible for activation of microglia, production of CXCL9, CXCL10, CCL8 and CCL12 and pathogenesis of ECM (Capuccini et al., 2016). Similarly, mice lacking IFN-I receptor $\left(\right.$ Ifnar $\left.1^{-/}\right)$had a significant decrease in inflammatory response with low levels of IL-6, CCL2, CCL3, CXCL1, CXCL10 and IFN- $\alpha$ and survived P. y. yoelii YM infection (Spaulding et al., 2016). IFNAR1 deficiency protected mice from ECM after $P$. berghei ANKA infection, and IFNAR1 signaling unleashed $\mathrm{CD} 8^{+} \mathrm{T}$ cell effector capacity that was crucial for ECM development (Ball et al., 2013). Deficiency of ubiquitin specific peptidase 15 (USP15), an IFN-I positive regulator, also protected mice from ECM and neuroinflammation (Torre et al., 2017). These studies, particularly $P$. berghei ANKA infections, suggest that IFN-I signaling, likely at days 3-6 pi, promotes inflammatory responses and ECM, and that high levels of IFN- $\gamma$, CXCL9, CXCL10, and $\mathrm{CD}^{+} \mathrm{T}$ cells likely contribute to the host death. On the other hand, the lethal parasite $P . y$. yoelii YM grows quickly in C57BL/6 mice with limited immune response before 
mouse death with high parasitemia ( $90 \%)$ on day 7 pi. High IFN-I levels $24 \mathrm{~h}$ pi in the P. y. yoelii YM-infected $\mathrm{Mavs}^{-1-}$ mice also lead to increased level of IFN- $\gamma$ and host survival, suggesting that increased inflammatory responses help control parasitemia and enhance host survival (Yu et al., 2016). Therefore, protection in terms of host survival depends on parasite/host models. If an infection induces a strong inflammatory response such as $P$. berghei ANKA infection, blockage of IFN-I signaling may reduce inflammation and enhance host survival. For an infection that does not induce a strong inflammatory response, higher levels of IFN-Is and IFN- $\gamma$ as well as an elevated inflammatory response may instead inhibit parasitemia and enhance survival. Because immune responses are dynamic, measurements of cytokines/chemokines and cell populations will vary at different time points during the course of infection, which can contribute to reports of contradictory results.

\section{IFN-I RESPONSES IN HUMAN INFECTIONS}

Studying IFN-I responses in human malaria infections have been mainly based on association of disease severity and/or IFN-I levels in the blood with gene expression level and/or genetic polymorphisms in genes playing a role in IFN-I responses. Microarray, RNA sequencing, single-cell sequencing, and genetic association can be important tools for studying IFN-I responses in human malaria infections. However, host immune responses, including IFN-I responses, will be highly variable among patients because the majority of clinical malaria infections in endemic regions carry multiple parasite strains with diverse genetic backgrounds (Conway et al., 1991; O’Brien et al., 2016; Zhu et al., 2019). Other factors such as the time of infection (usually unknown) and host genetic background will greatly influence the level and dynamics of IFN-I responses.

Consistent with observations in some studies using P. berghei ANKA infections (Vigario et al., 2001; Vigario et al., 2007), IFN-Is, particularly IFN- $\alpha$, appear to be protective in human infections of $P$. falciparum. The pre-antimalarial treatment levels of IFN- $\alpha$ were significantly lower in children with severe malaria than those with mild malaria (Luty et al., 2000). Similarly, lower circulating IFN- $\alpha$ was observed in children with severe malaria anemia (SMA) in Kenya, and two polymorphisms in IFN- $\alpha$ promoters [IFN- $\alpha 2$ (A173T) and IFN- $\alpha 8$ (T884A)] leading to reduced IFN- $\alpha$ production were associated with increased susceptibility to SMA and mortality (Kempaiah et al., 2012). Mild P. falciparum malaria following an episode of severe malaria was reported to be associated with induction of the IFN-I pathway in Malawian Children (Krupka et al., 2012). Patients with mild P. falciparum malaria in Rwanda had upregulated levels of IFN-Is, IFN- $\gamma$, complement system components, and nitric oxide (Subramaniam et al., 2015). These observations suggest a protective role of IFN-Is in malaria disease severity.

In contrast, IFN-I responses have also been associated with immune suppression and severe diseases in human malaria infections. Association between IFNAR1 variants and CM in children from Africa was observed; variants with lower IFNAR1 expression were associated with protection, whereas variants with increased IFNAR1 expression were associated with CM (Aucan et al., 2003; Khor et al., 2007; Ball et al., 2013; Feintuch et al., 2018). This is consistent with the observations that blocking IFNAR signaling can protect infected mice from severe disease symptoms. However, the dynamics of IFN-I levels during infection are unknown. Association between IFNAR1 and malaria susceptibility was also observed in Indian populations (Kanchan et al., 2015). But how these IFNAR1 variants regulate IFN-I responses to affect CM development requires further investigation. Dual transcriptome analyses of the host and parasite genes on samples from 46 malaria-infected Gambian children showed that disease severity was associated with increased expression of granulopoiesis and interferon- $\gamma$-related genes as well as with inadequate suppression of IFN-I signaling genes (Lee et al., 2018).

\section{POTENTIAL EXPLANATIONS FOR THE CONFLICTING ROLES OF IFN-IS}

IFN-I signaling can be protective leading to suppression of parasitemia and better survival or can inhibit DC and T cell activation to dampen adaptive response during malaria parasite infections. The effects or mechanisms of IFN-Is on parasitemia control and disease severity are complex, depending on both species of parasites and their hosts (parasite and host genetics), the timing and levels of IFN-I production, and possibly the subtypes of IFN-Is. Although the mechanisms of IFN-I in regulating host responses to infections of different malaria parasite species are diverse, some major themes can be established (Figure 3 and Supplementary Table S1). IFN-Is are protective if high levels of IFN-Is are produced early ( $24 \mathrm{~h} \mathrm{pi)}$ during an infection. P. berghei ANKA-infected C57BL/6 mice injected with IFN- $\alpha$ had significantly $(P<0.05)$ higher levels of IFN- $\gamma(772 \pm 73 \mathrm{pg} / \mathrm{ml}$, day $9 \mathrm{pi})$ than those receiving diluent $(180 \pm 14 \mathrm{pg} / \mathrm{ml})$, reduced parasitemia, and better host survival (Vigario et al., 2007). Elevated levels of IFN-Is (300-500 mg/ml IFN- $\alpha$ ) can reduce early (day 6) parasitemia as seen in P. $y$. nigeriensis N67 and $P$. chabaudi AS infections (Voisine et al., 2010; Wu et al., 2014; Wu et al., 2020). In another study, the effect of Ifnar1 $1^{-1-}$ on P. chabaudi AS parasitemia may not be significant, but the day 6 parasitemia is higher in the Ifnarl ${ }^{-1-}$ than those of WT mice (Kim et al., 2012). The protection mechanisms for these infections are largely unknown, probably associated with increased IFN- $\gamma$ expression at later stages of infection. Additionally, the levels of IFN- $\gamma$ and IL- 6 were also higher in mice with elevated early IFN-Is than infections with low IFN-Is, suggesting that IFN- $\gamma$, IL- 6 and other cytokines may contribute to the reduction of parasitemia and better host survival (Yu et al., 2016; Wu et al., 2020). In another model, $P$. y. yoelii YM infection of STING or MAVS deficient mice produced much higher peaks of IFN- $\alpha / \beta(\sim 2,500 \mathrm{pg} / \mathrm{ml}$ IFN- $\alpha$ and $\sim 1,200 \mathrm{pg} / \mathrm{ml}$ IFN- $\beta$ in MAVS knockout mice) than those observed in WT mice infected with $P . y$. nigeriensis N67 infection $24 \mathrm{~h}$ pi, which may provide stronger inhibition of parasite 
growth, leading to higher survival rates (Yu et al., 2016). In all the cases, IFN-I levels were quickly downregulated to background level after $24 \mathrm{~h}$ pi. These observations suggest a positive linkage between early peaks of IFN-Is and parasitemia control. It remains to be determined why $P$. $y$. nigeriensis N67 infection induces an early IFN-I responses, whereas other parasites such as $P$. y. nigeriensis $\mathrm{N} 67 \mathrm{C}$ and $P$. y. yoelii $\mathrm{YM}$ infections stimulate only low levels of IFN-Is ( $\sim 60 \mathrm{pg} / \mathrm{ml}$ IFN- $\alpha$ and almost no IFN- $\beta$ in YM infection) in C57BL/6 mice. Additionally, the molecular mechanism of downregulation of the $24 \mathrm{~h}$ IFN-I peak is still unknown.

On the other hand, blockage of IFN-I signaling has also been shown to increase DC and T cell activation and inflammation, which can promote survival or mortality depending on parasite species or strains. IFN-Is can cause immunopathology in acute viral infections (Davidson et al., 2014) and mediate immunosuppression during chronic viral infections (Teijaro et al., 2013; Wilson et al., 2013). Therefore, chronically elevated IFN-Is may explain the observations of immune suppression in various reports of malaria infections. In one study showing inhibition of DC and T cell activation by IFN-I signaling, Ifnarl ${ }^{-/-}$mice or multiple injections of anti-IFNAR antibodies were used (Zander et al., 2016), which is different from infection of wild type mice. In another study, infection of C57BL/6J mice with P. y. yoelii YM produced low level of IFN- $\alpha(20-70 \mathrm{pg} / \mathrm{ml})$, as reported in other studies (Yu et al., 2016; Wu et al., 2020); however, infection of Sting ${ }^{\mathrm{Gt} / \mathrm{Gt}}$ mice did not produce high levels of IFN-Is as observed in the study of ( $Y u$ et al., 2016). The reason for the discrepancy is unknown. Some possibilities include the uses of different sources of mice and parasite strains. Similarly, low levels of IFN- $\alpha / \beta$ were also observed in P. berghei ANKA-infected WT C57BL/6 mice $24 \mathrm{~h}$ pi (Haque et al., 2011; Haque et al., 2014). Again, depletion or blockage of IFN-I signaling resulted in higher levels of IFN $-\gamma$, better parasite control and improved host survival (Haque et al., 2011; Haque et al., 2014). Interestingly, higher levels of IFN- $\alpha$ were observed in wild type mice $48 \mathrm{~h}$ pi ( $\sim 70 \mathrm{pg} / \mathrm{ml}$ IFN- $\alpha$ vs $\sim 20 \mathrm{pg} / \mathrm{ml}$ at $24 \mathrm{~h})$ and day 4

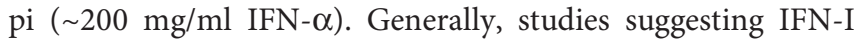
suppression of $\mathrm{T}$ cell activation or enhancement of inflammatory chemokines/cytokines used Ifnar $1^{-/-}$mice or multiple injections of anti-IFNAR antibodies, which are different from infections having a short time increase in IFN-I level. In these models, the absence of IFN-I signaling due to deficiency of IFNAR or antibody blockage may prompt the host to increase IFN-I production through autocrine feedback mechanisms, leading to activation and/or inhibition of alternative immune pathways that would not occur in infections with normal IFN-I signaling. Indeed, it has been shown that production of IFN- $\alpha / \beta$ early ( $24 \mathrm{~h}$ pi) during LCMV infection of mice was dependent upon the IFN- $\alpha / \beta \mathrm{R}$ and STAT1, and in the absence of IFN- $\alpha / \beta \mathrm{R}$ and the STAT1, an alternative delayed pathway dependent on a functional IFN- $\gamma \mathrm{R}$ was activated to produce IFN- $\alpha / \beta$ at $48 \mathrm{~h}$ pi (Malmgaard, 2004). Additionally, the alternative pathway for induction of IFN- $\alpha / \beta$ exists in IFN- $\alpha / \beta \mathrm{R}^{-/-}$ and $\mathrm{STAT}^{-/-}$mice but is absent in IFN- $\gamma \mathrm{R}^{-/-}$mice, suggesting that IFN- $\gamma$ was involved in the alternative pathway. Similar alternative pathways could exist in the Plasmodium-infected Ifnar ${ }^{-/-}$mice. However, no systematic measurement of IFN-I levels during infection were done in the studies using Ifnar $1^{-1-}$ mice or injections of anti-IFNAR antibodies. If the feedback alternative pathways of IFN-I responses exist in the infected Ifnar $1^{-/-}$mice, then high IFN-I levels may still play a role in protection through some unknown/alternative mechanisms. Measurements of the dynamic IFN-I levels during malaria infections will be important in order to understand the roles of IFN-Is in malaria infections.

In summary, an early IFN-I response ( $24 \mathrm{~h}$ pi) can be protective through suppression of parasitemia. Disruption of IFN-I signaling through IFNAR deficiency can be beneficial to the host; however, the levels of IFN-Is during the infections are mostly unknown. Whether elevated levels of IFN-Is are protective against severe diseases likely depends on parasite species/strains and host genetic background. It appears that all the protective effects are mediated through adequate levels of IFN- $\gamma$, proper activation of immune cells, and production of antibodies. How an early IFN-I response or the absence of IFN-I signaling regulates IFN- $\gamma$ and antibody responses during malaria parasite infections require additional investigations.

\section{REGULATION OF IFN-I RESPONSES DURING MALARIA PLASMODIUM INFECTIONS}

IFN-Is regulate many arms of the host immune responses; chronically elevated IFN-Is can suppress adaptive immunity and may also promote autoimmune diseases (Chen et al., 2020). Therefore, the levels of IFN-Is are also closely regulated by various mechanisms such as ubiquitination, phosphorylation and ADP-ribosylation of molecules in the IFN-I response pathways (Takaoka and Yamada, 2019; Zheng and Gao, 2020). Many molecules have been shown to amplify IFN-I signaling, including induction of STAT1 and IRF9 expression by IFN- $\gamma$ and interleukin6 (IL-6) (Ivashkiv and Donlin, 2014). There are also proteins such as suppressor of cytokine signaling 1 and 3 (SOCS1 and SOCS3) and ubiquitin carboxy-terminal hydrolase 18 (USP18) or microRNAs (miRNAs) that can suppress IFN-I responses (Yoshimura et al., 2007; Sarasin-Filipowicz et al., 2009). Because IFN-I responses during malaria infections shared the same pathways as those of viral infections, it can be expected that many regulatory mechanisms of IFN-I production in malaria parasite and virus infections are similar. However, malaria parasites are more complex organisms than viruses, and as a result, IFN-I responses and regulation during malaria parasite infections are likely more sophisticated than those of viruses.

All IFN-I response pathways use downstream transcription factors such as IRFs that regulate gene expression of IFNs. Using IRF deficient mice, many of these IRFs have been shown to play important roles in parasitemia control, disease severity, and ECM symptoms (Gun et al., 2014). Three single nucleotide polymorphisms (SNPs) in the IRF1 gene were shown to be correlated with blood parasite levels in malaria patients from west African, and one SNP (rs10065633) was associated with severe disease (Mangano et al., 2008). However, the molecular 
mechanisms regulating IFN-I responses during human malaria infections remain largely unknown. Recently, several studies using $P$. yoelii parasites begin to shed light on the mechanisms of IFN-I response regulation. A large number of host genes that interact with many genetic loci on the 14 chromosomes of $P$. yoelii, including clusters of genes involved in IFN-I responses, were identified using ts-eQTL (Wu et al., 2015a). Among the genes identified, a set of randomly selected putative ISGs (Ak3, Fosl1, Inpp4a, Havcr2, Fcgr1, Bc016423, S1pr5, Parp14, Satb1, Selenbp2, Helb, Helz2, and Lrp12) were found to inhibit luciferease signals driven by IFN- $\beta$ promoter, suggesting being negative regulators of the IFN-I signaling pathways (Figure 2). As mentioned above, P.y. nigeriensis N67 infection stimulates peaks of IFN- $\alpha / \beta$ approximately $24 \mathrm{~h}$ pi, and the IFN-I level quickly declines to background level soon after. These negative regulators likely play a role in the decline of the IFN-I levels. Selected genes from this study were further functionally characterized in vivo and in vitro. For example, CD40 (or TNF receptor superfamily member 5, TNFRSF5), a receptor expressed on the surfaces of many cell types, was shown to enhance STING protein level and STING-mediated IFN-I responses by affecting STING ubiquitination (Yao et al., 2016). On the other hand, the FOS-like antigen 1 (FOSL1), known as a component of FOS transcription factor, was shown to act as a negative regulator of IFN-I responses (Cai et al., 2017). After poly(I:C) or iRBC stimulation, FOSL1 translocated from the nucleus to the cytoplasm, where it interacted with TRAF3 and TRIF to reduce IRF3 phosphorylation and IFN-I production (Figure 2). Therefore, FOSL1 acts as a negative regulator of IFN-I response as well as a transcription factor, depending on its cellular location (Cai et al., 2017). Receptor transporter protein 4 gene (Rtp4) gene, another gene identified from the same tseQTL analysis, was clustered with ISGs such as Oas2, Dhx58, Ifit3, Usp18, Isg15, and Ifi35 (Wu et al., 2015a). RTP4 is induced by IFN-Is and binds to the TBK1 complex where it negatively regulates TBK1 signaling by interfering with the expression and phosphorylation of both TBK1 and IRF3 (He et al., 2020). Interestingly, RTP4 may have a specific role in brain pathology because mice deficient of RTP4 have lower West Nile virus titers in the brain and reduced hemorrhages in the cerebellum of $P$. berghei ANKA-infected mice compared with those of WT mice. Using the same ts-eQTL approach but with mRNAs collected $24 \mathrm{~h}$ after infection, a gene called March1, a member of the membrane-associated ring- $\mathrm{CH}$-type finger 1 family, was also found to be clustered with ISGs (Oas1d and Isg20) (Wu et al., 2020). MARCH1 was shown to interact with STING, MAVS, and various regulators of the IFN-I pathways to regulate IFN responses likely through protein ubiquitination. March1 $1^{-1-}$ mice infected with $P . y$. nigeriensis N67 or P. y. yoelii YM had significantly reduced serum IFN-I levels day 1 pi, but had increased number of $\mathrm{CD} 86^{+} \mathrm{DC}$ and elevated levels of IFN- $\gamma$ day $4 \mathrm{pi}$, and survived longer than WT mice. The results suggest that MARCH1 is an important immune regulator affecting IFN-I responses, $\mathrm{T}$ cell activation, and adaptive immunity. Studying malaria infections also reveals a cross regulation mechanism of two different IFN-I response pathways (Figure 2). It was found that STING- and MAVS-mediated low level IFN-I responses induced the negative regulator SOCS1 that in turn inhibited the more powerful TLR7/MYD88-mediated IFN-I responses in pDCs during P. y. yoelii YM blood stage infection (Yu et al., 2016). Additionally, activation of the AIM2, NLRP3 or adaptor caspase-1 inflammsome pathways enhances IL-1 $\beta$-mediated MYD88-TRAF3-IRF3 signaling and upregulation of SOCS1 that can inhibit MYD88-IRF7 signaling and IFN-I production in pDCs (Yu et al., 2018). These studies identify several regulators and mechanisms of IFN-I responses during malaria infections, which also contributes to our understanding of IFN-I signaling and regulation in general.

Molecules from malaria parasites may also modulate host IFN-I responses, although little is known about the mechanism of immune modulation by the parasites. A genetic locus on chromosome 13 of $P$. yoelii was significantly linked to expression of a large number ISGs, suggesting one or more parasite genes in the lcous may influnce host IFN-I responses (Wu et al., 2015a). Additionally, a C741Y amino acid substitution in the P. yoelii erythrocyte binding-like (PyEBL) protein appears to affect host IFN-I responses. The parasite carrying 741Y PyEBL (P. $y$. nigeriensis N67) stimulates higher levels of early IFN-Is than $P$. y. nigeriensis N67C that has 741C PyEBL (Peng et al., 2020; Wu et al., 2020). The substitution also altered host transcritomic responses, including genes in IFN-I responses, $\mathrm{T}$ helper cell activation, and IgG isotype switching (Peng et al., 2020). These observations indicate that parasite molecules can also modulate host IFN-I responses. However, parasite molecules that can stimulate and regulate IFN-I responses remain largely unknown.

\section{CONCLUSIONS}

Malaria parasite infection stimulates complex and differentially regulated immune responses, dependent on parasite and host species or strains. The parasite PAMPs and DAMPs are recognized by a number of host receptors. Asymptomatic liver stage infection induces the IFN-I responses, which reaches its peak during late liver stage when the parasite undergoes massive replication. The IFN-I signaling recruits and promotes the immune cells to control the liver stage infection. Parasite RNA appears to be the major ligand to elicit IFN-I responses in the hepatocytes, although parasite DNA may also stimulate IFN-I responses at this stage. It would be interesting to investigate whether parasite DNA and other host sensors are involved in the liver stage IFN-I response. After entering the bloodstream, sporozoites are phagocytized by DCs, which may trigger IFN-I responses in these DCs and lead to a positive feedback loop of IFN-I production. IFN-Is have been shown to be protective as well as deleterious in the blood stage parasite infections. Elevated level of IFN-Is or injection of IFN-Is can lead to the clearance of parasites and/or improved host survival rate. Parasite clearance could be mediated by phagocytosis and/or lysis of iRBCs activated by IFN-I signaling. However, deficiency in IFN-I signaling may also enhance $\mathrm{DC}$ function, $\mathrm{T}$ cell activation, and adaptive immune responses, which may reduce or aggravate disease severity depending on parasite strains and host genetic background. If an infection 
causes strong inflammatory response with severe disease symptoms (such as P. y. nigeriensis N67C and P. berghei ANKA), persistent elevated IFN-I levels may help alleviate disease symptoms by inhibiting $\mathrm{T}$ cell activation and suppressing inflammatory responses. Additionally, in many cases of rodent malaria infections (such as P. y. yoelii YM infection), the IFN-I levels are low, possibly due to mechanisms of immune evasion or inhibition of immune responses. An early strong IFN-I response can help suppress the rapid parasite growth, buying time for the host to develop protective immunity. Therefore, the roles of IFN-Is in malaria protection are dependent on parasite strains, host genetic background, and the level and timing of IFN-I production.

\section{AUTHOR CONTRIBUTIONS}

XH, LX, KT, JW, and X-ZS wrote the manuscript. JW and X-ZS conceived and designed the paper. JW created Figure 1. XH created Figure 2 and Figure 3. All authors contributed to the article and approved the submitted version.

\section{REFERENCES}

Angulo, I., and Fresno, M. (2002). Cytokines in the pathogenesis of and protection against malaria. Clin. Diagn. Lab. Immunol. 9 (6), 1145-1152. doi: 10.1128/ cdli.9.6.1145-1152.2002

Anstey, N. M., Douglas, N. M., Poespoprodjo, J. R., and Price, R. N. (2012). Plasmodium vivax: clinical spectrum, risk factors and pathogenesis. Adv. Parasitol. 80, 151-201. doi: 10.1016/B978-0-12-397900-1.00003-7

Arese, P., and Schwarzer, E. (1997). Malarial pigment (haemozoin): a very active 'inert' substance. Ann. Trop. Med. Parasitol. 91 (5), 501-516. doi: 10.1080/ 00034989760879

Ataide, M. A., Andrade, W. A., Zamboni, D. S., Wang, D., Souza Mdo, C., Franklin, B. S., et al. (2014). Malaria-induced NLRP12/NLRP3-dependent caspase-1 activation mediates inflammation and hypersensitivity to bacterial superinfection. PloS Pathog. 10 (1), e1003885. doi: 10.1371/journal.ppat.1003885

Aucan, C., Walley, A. J., Hennig, B. J., Fitness, J., Frodsham, A., Zhang, L., et al. (2003). Interferon-alpha receptor-1 (IFNAR1) variants are associated with protection against cerebral malaria in the Gambia. Genes Immun. 4 (4), 275282. doi: $10.1038 /$ sj.gene.6363962

Babatunde, K. A., Mbagwu, S., Hernandez-Castaneda, M. A., Adapa, S. R., Walch, M., Filgueira, L., et al. (2018). Malaria infected red blood cells release small regulatory RNAs through extracellular vesicles. Sci. Rep. 8, 884. doi: 10.1038/ s41598-018-19149-9

Babatunde, K. A., Subramanian, B. Y., Ahouidi, A. D., Murillo, P. M., Walch, M., and Mantel, P. Y. (2020). Role of Extracellular Vesicles in Cellular Cross Talk in Malaria. Front. Immunol. 11, 22. doi: 10.3389/fimmu.2020.00022

Baccarella, A., Fontana, M. F., Chen, E. C., and Kim, C. C. (2013). Toll-Like Receptor 7 Mediates Early Innate Immune Responses to Malaria. Infect. Immun. 81 (12), 4431-4442. doi: 10.1128/Iai.00923-13

Ball, E. A., Sambo, M. R., Martins, M., Trovoada, M. J., Benchimol, C., Costa, J., et al. (2013). IFNAR1 controls progression to cerebral malaria in children and CD8+ T cell brain pathology in Plasmodium berghei-infected mice. J. Immunol. 190 (10), 5118-5127. doi: 10.4049/jimmunol.1300114

Barrera, V., Skorokhod, O. A., Baci, D., Gremo, G., Arese, P., and Schwarzer, E. (2011). Host fibrinogen stably bound to hemozoin rapidly activates monocytes via TLR-4 and CD11b/CD18-integrin: a new paradigm of hemozoin action. Blood 117 (21), 5674-5682. doi: 10.1182/blood-2010-10-312413

Barton, G. M., and Kagan, J. C. (2009). A cell biological view of Toll-like receptor function: regulation through compartmentalization. Nat. Rev. Immunol. 9 (8), 535-542. doi: 10.1038/nri2587

Beiting, D. P. (2014). Protozoan parasites and type I interferons: a cold case reopened. Trends Parasitol. 30 (10), 491-498. doi: 10.1016/j.pt.2014.07.007

\section{FUNDING}

This work was supported by the Division of Intramural Research, National Institute of Allergy and Infectious Diseases (NIAID), National Institutes of Health (NIH), USA.

\section{ACKNOWLEDGMENTS}

We thank Diane Cooper, MS, of the NIH library for manuscript editing assistance.

\section{SUPPLEMENTARY MATERIAL}

The Supplementary Material for this article can be found online at: https://www.frontiersin.org/articles/10.3389/fcimb.2020.594621/ full\#supplementary-material

Belgnaoui, S. M., Paz, S., and Hiscott, J. (2011). Orchestrating the interferon antiviral response through the mitochondrial antiviral signaling (MAVS) adapter. Curr. Opin. Immunol. 23 (5), 564-572. doi: 10.1016/j.coi.2011.08.001

Beutler, B. A. (2009). TLRs and innate immunity. Blood 113 (7), 1399-1407. doi: 10.1182/blood-2008-07-019307

Borden, E. C., Sen, G. C., Uze, G., Silverman, R. H., Ransohoff, R. M., Foster, G. R., et al. (2007). Interferons at age 50: past, current and future impact on biomedicine. Nat. Rev. Drug Discov. 6 (12), 975-990. doi: 10.1038/nrd2422

Boxx, G. M., and Cheng, G. (2016). The Roles of Type I Interferon in Bacterial Infection. Cell Host Microbe 19 (6), 760-769. doi: 10.1016/j.chom.2016.05.016

Brown, D., and Waneck, G. L. (1992). Glycosyl-phosphatidylinositol-anchored membrane proteins. J. Am. Soc. Nephrol. 3 (4), 895-906.

Brubaker, S. W., Bonham, K. S., Zanoni, I., and Kagan, J. C. (2015). Innate immune pattern recognition: a cell biological perspective. Annu. Rev. Immunol. 33, 257-290. doi: 10.1146/annurev-immunol-032414-112240

Bui, T. M., Wiesolek, H. L., and Sumagin, R. (2020). ICAM-1: A master regulator of cellular responses in inflammation, injury resolution, and tumorigenesis. J. Leukoc. Biol. 108 (3), 787-799. doi: 10.1002/JLB.2MR0220-549R

Butler, N. S., Schmidt, N. W., Vaughan, A. M., Aly, A. S., Kappe, S. H., and Harty, J. T. (2011). Superior antimalarial immunity after vaccination with late liver stage-arresting genetically attenuated parasites. Cell Host Microbe 9 (6), 451462. doi: 10.1016/j.chom.2011.05.008

Cai, B., Wu, J., Yu, X., Su, X. Z., and Wang, R. F. (2017). FOSL1 Inhibits Type I Interferon Responses to Malaria and Viral Infections by Blocking TBK1 and TRAF3/TRIF Interactions. mBio 8 (1), e02161-16. doi: 10.1128/mBio.02161-16

Campos, M. A., Almeida, I. C., Takeuchi, O., Akira, S., Valente, E. P., Procopio, D. O., et al. (2001). Activation of Toll-like receptor-2 by glycosylphosphatidylinositol anchors from a protozoan parasite. J. Immunol. 167 (1), 416-423. doi: 10.4049/jimmunol.167.1.416

Capuccini, B., Lin, J., Talavera-Lopez, C., Khan, S. M., Sodenkamp, J., Spaccapelo, R., et al. (2016). Transcriptomic profiling of microglia reveals signatures of cell activation and immune response, during experimental cerebral malaria. Sci. Rep. 6:39258. doi: 10.1038/srep39258

Carrero, J. A. (2013). Confounding roles for type I interferons during bacterial and viral pathogenesis. Int. Immunol. 25 (12), 663-669. doi: 10.1093/intimm/dxt050

Celada, A., Cruchaud, A., and Perrin, L. H. (1983). Phagocytosis of Plasmodium falciparum-parasitized erythrocytes by human polymorphonuclear leukocytes. J. Parasitol. 69 (1), 49-53. doi: 10.2307/3281273

Chen, H. J., Tas, S. W., and de Winther, M. P. J. (2020). Type-I interferons in atherosclerosis. J. Exp. Med. 217 (1), e20190459. doi: 10.1084/jem.20190459

Chiu, Y. H., MacMillan, J. B., and Chen, Z. J. J. (2009). RNA Polymerase III Detects Cytosolic DNA and Induces Type I Interferons through the RIG-I Pathway. Cell 138 (3), 576-591. doi: 10.1016/j.cell.2009.06.015 
Clark, I. A., Alleva, L. M., Budd, A. C., and Cowden, W. B. (2008). Understanding the role of inflammatory cytokines in malaria and related diseases. Travel Med. Infect. Dis. 6 (1-2), 67-81. doi: 10.1016/j.tmaid.2007.07.002

Coch, C., Hommertgen, B., Zillinger, T., Dassler-Plenker, J., Putschli, B., Nastaly, M., et al. (2019). Human TLR8 Senses RNA From Plasmodium falciparumInfected Red Blood Cells Which Is Uniquely Required for the IFN-gamma Response in NK Cells. Front. Immunol. 10, 371. doi: 10.3389/ fimmu.2019.00371

Combes, V., Simon, A. C., Grau, G. E., Arnoux, D., Camoin, L., Sabatier, F., et al. (1999). In vitro generation of endothelial microparticles and possible prothrombotic activity in patients with lupus anticoagulant. J. Clin. Invest. 104 (1), 93-102. doi: 10.1172/Jci4985

Combes, V., Coltel, N., Alibert, M., van Eck, M., Raymond, C., Juhan-Vague, I., et al. (2005). ABCA1 gene deletion protects against cerebral malaria - Potential pathogenic role of microparticles in neuropathology. Am. J. Pathol. 166 (1), 295-302. doi: 10.1016/S0002-9440(10)62253-5

Conway, D. J., Greenwood, B. M., and McBride, J. S. (1991). The epidemiology of multiple-clone Plasmodium falciparum infections in Gambian patients. Parasitol 103 Pt 1, 1-6. doi: 10.1017/s0031182000059217

Couper, K. N., Barnes, T., Hafalla, J. C. R., Combes, V., Ryffel, B., Secher, T., et al. (2010). Parasite-Derived Plasma Microparticles Contribute Significantly to Malaria Infection-Induced Inflammation through Potent Macrophage Stimulation. PloS Pathog. 6 (1), e1000744. doi: 10.1371/journal.ppat.1000744

Crompton, P. D., Moebius, J., Portugal, S., Waisberg, M., Hart, G., Garver, L. S., et al. (2014). Malaria immunity in man and mosquito: insights into unsolved mysteries of a deadly infectious disease. Annu. Rev. Immunol. 32, 157-187. doi: 10.1146/annurev-immunol-032713-120220

Cui, S., Eisenacher, K., Kirchhofer, A., Brzozka, K., Lammens, A., Lammens, K., et al. (2008). The C-terminal regulatory domain is the RNA 5 '-triphosphate sensor of RIG-I. Mol. Cell 29 (2), 169-179. doi: 10.1016/j.molcel.2007.10.032

Davidson, S., Crotta, S., McCabe, T. M., and Wack, A. (2014). Pathogenic potential of interferon alphabeta in acute influenza infection. Nat. Commun. 5, 3864. doi: $10.1038 /$ ncomms4864

deWalick, S., Amante, F. H., McSweeney, K. A., Randall, L. M., Stanley, A. C., Haque, A., et al. (2007). Cutting edge: conventional dendritic cells are the critical APC required for the induction of experimental cerebral malaria. J. Immunol. 178 (10), 6033-6037. doi: 10.4049/jimmunol.178.10.6033

Dostert, C., Guarda, G., Romero, J. F., Menu, P., Gross, O., Tardivel, A., et al. (2009). Malarial hemozoin is a Nalp3 inflammasome activating danger signal. PloS One 4 (8), e6510. doi: 10.1371/journal.pone.0006510

Dunst, J., Kamena, F., and Matuschewski, K. (2017). Cytokines and Chemokines in Cerebral Malaria Pathogenesis. Front. Cell Infect. Microbiol. 7:324. doi: $10.3389 /$ fcimb.2017.00324

El-Assaad, F., Wheway, J., Hunt, N. H., Grau, G. E. R., and Combes, V. (2014). Production, Fate and Pathogenicity of Plasma Microparticles in Murine Cerebral Malaria. PloS Pathog. 10 (3), e1003839. doi: 10.1371/journal.ppat.1003839

Emran, T. B., Iyori, M., Ono, Y., Amelia, F., Yusuf, Y., Islam, A., et al. (2018). Baculovirus-Induced Fast-Acting Innate Immunity Kills Liver-Stage Plasmodium. J. Immunol. 201 (8), 2441-2451. doi: 10.4049/jimmunol.1800908

Favre, N., Da Laperousaz, C., Ryffel, B., Weiss, N. A., Imhof, B. A., Rudin, W., et al. (1999). Role of ICAM-1 (CD54) in the development of murine cerebral malaria. Microbes Infect. 1 (12), 961-968. doi: 10.1016/s1286-4579(99)80513-9

Feintuch, C. M., Tare, A., Cusumano, L. R., Benayoun, J., Ryu, S., Sixpence, A., et al. (2018). Type I Interferon Receptor Variants in Gene Regulatory Regions are Associated with Susceptibility to Cerebral Malaria in Malawi. Am. J. Trop. Med. Hyg. 98 (6), 1692-1698. doi: 10.4269/ajtmh.17-0887

Ferguson, M. A. J., Brimacombe, J. S., Brown, J. R., Crossman, A., Dix, A., Field, R. A., et al. (1999). The GPI biosynthetic pathway as a therapeutic target for African sleeping sickness. Biochim. Biophys. Acta Mol. Basis Dis. 1455 (2-3), 327-340. doi: 10.1016/S0925-4439(99)00058-7

Fontana, M. F., de Melo, G. L., Anidi, C., Hamburger, R., Kim, C. Y., Lee, S. Y., et al. (2016). Macrophage Colony Stimulating Factor Derived from CD4+ T Cells Contributes to Control of a Blood-Borne Infection. PloS Pathog. 12 (12), e1006046. doi: 10.1371/journal.ppat.1006046

Freeman, R. R., and Parish, C. R. (1981). Plasmodium yoelii: antibody and the maintenance of immunity in BALB/c mice. Exp. Parasitol. 52 (1), 18-24. doi: 10.1016/0014-4894(81)90056-4
Freyssinet, J. M. (2003). Cellular microparticles: what are they bad or good for? J. Thromb. Haemost. 1 (7), 1655-1662. doi: 10.1046/j.1538-7836.2003.00309.x

Gallego-Marin, C., Schrum, J. E., Andrade, W. A., Shaffer, S. A., Giraldo, L. F., Lasso, A. M., et al. (2018). Cyclic GMP-AMP Synthase Is the Cytosolic Sensor of Plasmodium falciparum Genomic DNA and Activates Type I IFN in Malaria. J. Immunol. 200 (2), 768-774. doi: 10.4049/jimmunol.1701048

Gazzinelli, R. T., Kalantari, P., Fitzgerald, K. A., and Golenbock, D. T. (2014). Innate sensing of malaria parasites. Nat. Rev. Immunol. 14 (11), 744-757. doi: $10.1038 /$ nri3742

Gotz, A., Tang, M. S., Ty, M. C., Arama, C., Ongoiba, A., Doumtabe, D., et al. (2017). Atypical activation of dendritic cells by Plasmodium falciparum. Proc. Natl. Acad. Sci. U.S.A. 114 (49), E10568-E10577. doi: 10.1073/pnas.1708383114

Gowda, N. M., Wu, X. Z., and Gowda, D. C. (2011). The Nucleosome (HistoneDNA Complex) Is the TLR9-Specific Immunostimulatory Component of Plasmodium falciparum That Activates DCs. PloS One 6 (6), e20398. doi: 10.1371/journal.pone.0020398

Grangeiro de Carvalho, E., Bonin, M., Kremsner, P. G., and Kun, J. F. (2011). Plasmodium falciparum-infected erythrocytes and IL-12/IL-18 induce diverse transcriptomes in human NK cells: IFN-alpha/beta pathway versus TREM signaling. PloS One 6 (9), e24963. doi: 10.1371/journal.pone.0024963

Gui, X., Yang, H., Li, T., Tan, X., Shi, P., Li, M., et al. (2019). Autophagy induction via STING trafficking is a primordial function of the cGAS pathway. Nature 567 (7747), 262-266. doi: 10.1038/s41586-019-1006-9

Gun, S. Y., Claser, C., Tan, K. S., and Renia, L. (2014). Interferons and interferon regulatory factors in malaria. Mediators Inflammation 2014:243713. doi: $10.1155 / 2014 / 243713$

Hahn, W. O., Butler, N. S., Lindner, S. E., Akilesh, H. M., Sather, D. N., Kappe, S. H., et al. (2018). cGAS-mediated control of blood-stage malaria promotes Plasmodium-specific germinal center responses. JCI Insight 3 (2), e94142. doi: 10.1172/jci.insight.94142

Haque, A., Best, S. E., Ammerdorffer, A., Desbarrieres, L., de Oca, M. M., Amante, F. H., et al. (2011). Type I interferons suppress CD4(+) T-cell-dependent parasite control during blood-stage Plasmodium infection. Eur. J. Immunol. 41 (9), 2688-2698. doi: 10.1002/eji.201141539

Haque, A., Best, S. E., Montes de Oca, M., James, K. R., Ammerdorffer, A., Edwards, C. L., et al. (2014). Type I IFN signaling in CD8- DCs impairs Th1dependent malaria immunity. J. Clin. Invest. 124 (6), 2483-2496. doi: 10.1172/ JCI70698

He, X., Ashbrook, A. W., Du, Y., Wu, J., Hoffmann, H. H., Zhang, C., et al. (2020). RTP4 inhibits IFN-I response and enhances experimental cerebral malaria and neuropathology. Proc. Natl. Acad. Sci. U.S.A. 117 (32), 19465-19474. doi: 10.1073/pnas.2006492117

Hirako, I. C., Gallego-Marin, C., Ataide, M. A., Andrade, W. A., Gravina, H., Rocha, B. C., et al. (2015). DNA-Containing Immunocomplexes Promote Inflammasome Assembly and Release of Pyrogenic Cytokines by CD14+ CD16 + CD64high CD32low Inflammatory Monocytes from Malaria Patients. mBio 6 (6), e01605-e01615. doi: 10.1128/mBio.01605-15

Ho, M., and Webster, H. K. (1989). Immunology of human malaria. A cellular perspective. Parasite Immunol. 11 (2), 105-116. doi: 10.1111/j.1365-3024.1989.tb00652.x

Hornung, V., and Latz, E. (2010). Intracellular DNA recognition. Nat. Rev. Immunol. 10 (2), 123-130. doi: 10.1038/nri2690

Hornung, V., Rothenfusser, S., Britsch, S., Krug, A., Jahrsdorfer, B., Giese, T., et al. (2002). Quantitative expression of toll-like receptor 1-10 mRNA in cellular subsets of human peripheral blood mononuclear cells and sensitivity to $\mathrm{CpG}$ oligodeoxynucleotides. J. Immunol. 168 (9), 4531-4537. doi: 10.4049/ jimmunol.168.9.4531

Hou, F. J., Sun, L. J., Zheng, H., Skaug, B., Jiang, Q. X., and Chen, Z. J. (2011). MAVS Forms Functional Prion-like Aggregates to Activate and Propagate Antiviral Innate Immune Response (vol 146, pg 448, 2011). Cell 146 (5), 841841. doi: 10.1016/j.cell.2011.08.013

Huang, K. Y., Schultz, W. W., and Gordon, F. B. (1968). Interferon induced by Plasmodium berghei. Science 162 (3849), 123-124. doi: 10.1126/science.162.3849.123 Isaacs, A., and Lindenmann, J. (1957). Virus interference. I. The interferon. Proc. R. Soc. Lond. B Biol. Sci. 147 (927), 258-267. doi: 10.1098/rspb.1957.0048

Isaacs, A., Lindenmann, J., and Valentine, R. C. (1957). Virus interference. II. Some properties of interferon. Proc. R. Soc. Lond. B Biol. Sci. 147 (927), 268273. doi: $10.1098 / \mathrm{rspb} .1957 .0049$ 
Ishikawa, H., and Barber, G. N. (2008). STING is an endoplasmic reticulum adaptor that facilitates innate immune signalling. Nature 455 (7213), 674-678. doi: $10.1038 /$ nature 07317

Ivashkiv, L. B., and Donlin, L. T. (2014). Regulation of type I interferon responses. Nat. Rev. Immunol. 14 (1), 36-49. doi: 10.1038/nri3581

Jacobs, J. L., and Coyne, C. B. (2013). Mechanisms of MAVS Regulation at the Mitochondrial Membrane. J. Mol. Biol. 425 (24), 5009-5019. doi: 10.1016/ j.jmb.2013.10.007

Jahiel, R. I., Nussenzweig, R. S., Vanderberg, J., and Vilcek, J. (1968a). Anti-malarial effect of interferon inducers at different stages of development of Plasmodium berghei in the mouse. Nature 220 (5168), 710-711. doi: 10.1038/220710a0

Jahiel, R. I., Vilcek, J., Nussenzweig, R., and Vanderberg, J. (1968b). Interferon inducers protect mice against plasmodium berghei malaria. Science 161 (3843), 802-804. doi: 10.1126/science.161.3843.802

Jahiel, R. I., Vilcek, J., and Nussenzweig, R. S. (1970). Exogenous interferon protects mice against Plasmodium berghei malaria. Nature 227 (5265), 13501351. doi: $10.1038 / 2271350 \mathrm{a} 0$

Jani, D., Nagarkatti, R., Beatty, W., Angel, R., Slebodnick, C., Andersen, J., et al. (2008). HDP-a novel heme detoxification protein from the malaria parasite. PloS Pathog. 4 (4), e1000053. doi: 10.1371/journal.ppat.1000053

Jaramillo, M., Bellemare, M. J., Martel, C., Shio, M. T., Contreras, A. P., Godbout, M., et al. (2009). Synthetic Plasmodium-like hemozoin activates the immune response: a morphology - function study. PloS One 4 (9), e6957. doi: 10.1371/ journal.pone.0006957

Jin, L., Getahun, A., Knowles, H. M., Mogan, J., Akerlund, L. J., Packard, T. A., et al. (2013). STING/MPYS mediates host defense against Listeria monocytogenes infection by regulating $\mathrm{Ly} 6 \mathrm{C}(\mathrm{hi})$ monocyte migration. J. Immunol. 190 (6), 2835-2843. doi: 10.4049/jimmunol.1201788

Jobe, O., Donofrio, G., Sun, G., Liepinsh, D., Schwenk, R., and Krzych, U. (2009). Immunization with radiation-attenuated Plasmodium berghei sporozoites induces liver cCD8alpha+DC that activate CD8+T cells against liver-stage malaria. PloS One 4 (4), e5075. doi: 10.1371/journal.pone.0005075

Johnstone, R. M., Adam, M., Hammond, J. R., Orr, L., and Turbide, C. (1987). Vesicle Formation during Reticulocyte Maturation - Association of PlasmaMembrane Activities with Released Vesicles (Exosomes). J. Biol. Chem. 262 (19), 9412-9420

Kaczmarek, A., Vandenabeele, P., and Krysko, D. V. (2013). Necroptosis: the release of damage-associated molecular patterns and its physiological relevance. Immunity 38 (2), 209-223. doi: 10.1016/j.immuni.2013.02.003

Kalantari, P., DeOliveira, R. B., Chan, J., Corbett, Y., Rathinam, V., Stutz, A., et al. (2014). Dual Engagement of the NLRP3 and AIM2 Inflammasomes by Plasmodium-Derived Hemozoin and DNA during Malaria. Cell Rep. 6 (1), 196-210. doi: 10.1016/j.celrep.2013.12.014

Kanchan, K., Jha, P., Pati, S. S., Mohanty, S., Mishra, S. K., Sharma, S. K., et al. (2015). Interferon-gamma (IFNG) microsatellite repeat and single nucleotide polymorphism haplotypes of IFN-alpha receptor (IFNAR1) associated with enhanced malaria susceptibility in Indian populations. Infect. Genet. Evol. 29, 6-14. doi: 10.1016/j.meegid.2014.10.030

Kawagoe, T., Sato, S., Matsushita, K., Kato, H., Matsui, K., Kumagai, Y., et al. (2008). Sequential control of Toll-like receptor-dependent responses by IRAK1 and IRAK2. Nat. Immunol. 9 (6), 684-691. doi: 10.1038/ni.1606

Kawai, T., and Akira, S. (2008). Toll-like receptor and RIG-I-like receptor signaling. Ann. N. Y. Acad. Sci. 1143, 1-20. doi: 10.1196/annals.1443.020

Kawai, T., and Akira, S. (2010). The role of pattern-recognition receptors in innate immunity: update on Toll-like receptors. Nat. Immunol. 11 (5), 373-384. doi: $10.1038 /$ ni. 1863

Kawai, T., and Akira, S. (2011). Toll-like Receptors and Their Crosstalk with Other Innate Receptors in Infection and Immunity. Immunity 34 (5), 637-650. doi: 10.1016/j.immuni.2011.05.006

Kawai, T., Takahashi, K., Sato, S., Coban, C., Kumar, H., Kato, H., et al. (2005). IPS-1, an adaptor triggering RIG-I- and Mda5-mediated type I interferon induction. Nat. Immunol. 6 (10), 981-988. doi: 10.1038/ni1243

Kawasaki, T., and Kawai, T. (2014). Toll-like receptor signaling pathways. Front. Immunol. 5:461. doi: 10.3389/fimmu.2014.00461

Kempaiah, P., Anyona, S. B., Raballah, E., Davenport, G. C., Were, T., Hittner, J. B., et al. (2012). Reduced interferon (IFN)-alpha conditioned by IFNA2 (-173) and IFNA8 (-884) haplotypes is associated with enhanced susceptibility to severe malarial anemia and longitudinal all-cause mortality. Hum. Genet. 131 (8), 1375-1391. doi: 10.1007/s00439-012-1175-1

Khor, C. C., Vannberg, F. O., Chapman, S. J., Walley, A., Aucan, C., Loke, H., et al. (2007). Positive replication and linkage disequilibrium mapping of the chromosome 21q22.1 malaria susceptibility locus. Genes Immun. 8 (7), 570576. doi: 10.1038/sj.gene.6364417

Kim, C. C., Nelson, C. S., Wilson, E. B., Hou, B., DeFranco, A. L., and DeRisi, J. L. (2012). Splenic red pulp macrophages produce type I interferons as early sentinels of malaria infection but are dispensable for control. PloS One 7 (10), e48126. doi: 10.1371/journal.pone.0048126

Kotenko, S. V. (2011). IFN-lambdas. Curr. Opin. Immunol. 23 (5), 583-590. doi: 10.1016/j.coi.2011.07.007

Krishnegowda, G., Hajjar, A. M., Zhu, J., Douglass, E. J., Uematsu, S., Akira, S., et al. (2005). Induction of proinflammatory responses in macrophages by the glycosylphosphatidylinositols of Plasmodium falciparum: cell signaling receptors, glycosylphosphatidylinositol (GPI) structural requirement, and regulation of GPI activity. J. Biol. Chem. 280 (9), 8606-8616. doi: 10.1074/ jbc.M413541200

Krupka, M., Seydel, K., Feintuch, C. M., Yee, K., Kim, R., Lin, C. Y., et al. (2012). Mild Plasmodium falciparum malaria following an episode of severe malaria is associated with induction of the interferon pathway in Malawian children. Infect. Immun. 80 (3), 1150-1155. doi: 10.1128/IAI.06008-11

Kurup, S. P., Anthony, S. M., Hancox, L. S., Vijay, R., Pewe, L. L., Moioffer, S. J., et al. (2019a). Monocyte-Derived CD11c(+) Cells Acquire Plasmodium from Hepatocytes to Prime CD8 T Cell Immunity to Liver-Stage Malaria. Cell Host Microbe 25 (4), 565-577 e566. doi: 10.1016/j.chom.2019.02.014

Kurup, S. P., Butler, N. S., and Harty, J. T. (2019b). T cell-mediated immunity to malaria. Nat. Rev. Immunol. 19 (7), 457-471. doi: 10.1038/s41577-019-0158-Z

Lamphier, M. S., Sirois, C. M., Verma, A., Golenbock, D. T., and Latz, E. (2006). TLR9 and the recognition of self and non-self nucleic acids. Oligonucleotide Ther. 1082, 31-43. doi: 10.1196/annals.1348.005

Langhorne, J., Simon-Haarhaus, B., and Meding, S. J. (1990). The role of CD4+ T cells in the protective immune response to Plasmodium chabaudi in vivo. Immunol. Lett. 25 (1-3), 101-107. doi: 10.1016/0165-2478(90)90099-c

Laulagnier, K., Motta, C., Hamdi, S., Roy, S., Fauvelle, F., Pageaux, J. F., et al. (2004). Mast cell- and dendritic cell-derived exosomes display a specific lipid composition and an unusual membrane organization. Biochem. J. 380 (Pt 1), 161-171. doi: 10.1042/BJ20031594

Le Bon, A., Schiavoni, G., D’Agostino, G., Gresser, I., Belardelli, F., and Tough, D. F. (2001). Type i interferons potently enhance humoral immunity and can promote isotype switching by stimulating dendritic cells in vivo. Immunity 14 (4), 461-470. doi: 10.1016/s1074-7613(01)00126-1

Lee, H. J., Georgiadou, A., Walther, M., Nwakanma, D., Stewart, L. B., Levin, M., et al. (2018). Integrated pathogen load and dual transcriptome analysis of systemic host-pathogen interactions in severe malaria. Sci. Transl. Med. 10 (447), eaar3619. doi: 10.1126/scitranslmed.aar3619

Liehl, P., and Mota, M. M. (2012). Innate recognition of malarial parasites by mammalian hosts. Int. J. Parasitol. 42 (6), 557-566. doi: 10.1016/j.ijpara.2012.04.006

Liehl, P., Zuzarte-Luis, V., Chan, J., Zillinger, T., Baptista, F., Carapau, D., et al. (2014). Host-cell sensors for Plasmodium activate innate immunity against liver-stage infection. Nat. Med. 20 (1), 47-53. doi: 10.1038/nm.3424

Liehl, P., Meireles, P., Albuquerque, I. S., Pinkevych, M., Baptista, F., Mota, M. M., et al. (2015). Innate immunity induced by Plasmodium liver infection inhibits malaria reinfections. Infect. Immun. 83 (3), 1172-1180. doi: 10.1128/IAI.02796-14

Loo, Y. M., and Gale, M. (2011). Immune Signaling by RIG-I-like Receptors. Immunity 34 (5), 680-692. doi: 10.1016/j.immuni.2011.05.003

Lu, Z. Y., Serghides, L., Patel, S. N., Degousee, N., Rubin, B. B., Krishnegowda, G., et al. (2006). Disruption of JNK2 decreases the cytokine response to Plasmodium falciparum glycosylphosphatidylinositol in vitro and confers protection in a cerebral malaria model. J. Immunol. 177 (9), 6344-6352. doi: 10.4049/jimmunol.177.9.6344

Lukhele, S., Boukhaled, G. M., and Brooks, D. G. (2019). Type I interferon signaling, regulation and gene stimulation in chronic virus infection. Semin. Immunol. 43, 101277. doi: 10.1016/j.smim.2019.05.001

Luty, A. J., Perkins, D. J., Lell, B., Schmidt-Ott, R., Lehman, L. G., Luckner, D., et al. (2000). Low interleukin-12 activity in severe Plasmodium falciparum malaria. Infect. Immun. 68 (7), 3909-3915. doi: 10.1128/iai.68.7.3909-3915.2000 
Malmgaard, L. (2004). Induction and regulation of IFNs during viral infections. J. Interferon Cytokine Res. 24 (8), 439-454. doi: 10.1089/1079990041689665

Mangano, V. D., Luoni, G., Rockett, K. A., Sirima, B. S., Konate, A., Forton, J., et al. (2008). Interferon regulatory factor-1 polymorphisms are associated with the control of Plasmodium falciparum infection. Genes Immun. 9 (2), 122-129. doi: $10.1038 /$ s.gene.6364456

Meylan, E., Curran, J., Hofmann, K., Moradpour, D., Binder, M., Bartenschlager, R., et al. (2005). Cardif is an adaptor protein in the RIG-I antiviral pathway and is targeted by hepatitis C virus. Nature 437 (7062), 1167-1172. doi: 10.1038/nature04193

Miller, J. L., Sack, B. K., Baldwin, M., Vaughan, A. M., and Kappe, S. H. I. (2014). Interferon-Mediated Innate Immune Responses against Malaria Parasite Liver Stages. Cell Rep. 7 (2), 436-447. doi: 10.1016/j.celrep.2014.03.018

Minkah, N. K., Wilder, B. K., Sheikh, A. A., Martinson, T., Wegmair, L., Vaughan, A. M., et al. (2019). Innate immunity limits protective adaptive immune responses against pre-erythrocytic malaria parasites. Nat. Commun. 10 (1), 3950. doi: 10.1038/s41467-019-11819-0

Mo, A. X., and McGugan, G. (2018). Understanding the Liver-Stage Biology of Malaria Parasites: Insights to Enable and Accelerate the Development of a Highly Efficacious Vaccine. Am. J. Trop. Med. Hyg. 99 (4), 827-832. doi: 10.4269/ajtmh.17-0895

Montes de Oca, M., Kumar, R., Rivera, F. L., Amante, F. H., Sheel, M., Faleiro, R. J., et al. (2016). Type I Interferons Regulate Immune Responses in Humans with Blood-Stage Plasmodium falciparum Infection. Cell Rep. 17 (2), 399-412. doi: 10.1016/j.celrep.2016.09.015

Morrell, C. N., Srivastava, K., Swaim, A., Lee, M. T., Chen, J., Nagineni, C., et al. (2011). Beta interferon suppresses the development of experimental cerebral malaria. Infect. Immun. 79 (4), 1750-1758. doi: 10.1128/IAI.00810-10

Murphy, J. R., and Lefford, M. J. (1979). Host defenses in murine malaria: evaluation of the mechanisms of immunity to Plasmodium yoelii infection. Infect. Immun. 23 (2), 384-391. doi: 10.1128/IAI.23.2.384-391.1979

Naik, R. S., Branch, O. H., Woods, A. S., Vijaykumar, M., Perkins, D. J., Nahlen, B. L., et al. (2000). Glycosylphosphatidylinositol anchors of Plasmodium falciparum: Molecular characterization and naturally elicited antibody response that may provide immunity to malaria pathogenesis. J. Exp. Med. 192 (11), 1563-1575. doi: 10.1084/jem.192.11.1563

Ng, C. T., Sullivan, B. M., Teijaro, J. R., Lee, A. M., Welch, M., Rice, S., et al. (2015). Blockade of interferon Beta, but not interferon alpha, signaling controls persistent viral infection. Cell Host Microbe 17 (5), 653-661. doi: 10.1016/j.chom.2015.04.005

Ng, C. T., Mendoza, J. L., Garcia, K. C., and Oldstone, M. B. (2016). Alpha and Beta Type 1 Interferon Signaling: Passage for Diverse Biologic Outcomes. Cell 164 (3), 349-352. doi: 10.1016/j.cell.2015.12.027

Nie, L., Cai, S. Y., Shao, J. Z., and Chen, J. (2018). Toll-Like Receptors, Associated Biological Roles, and Signaling Networks in Non-Mammals. Front. Immunol. 9:1523. doi: $10.3389 /$ fimmu. 2018.01523

Ning, S., Pagano, J. S., and Barber, G. N. (2011). IRF7: activation, regulation, modification and function. Genes Immun. 12 (6), 399-414. doi: 10.1038/gene.2011.21

Nolte-'t Hoen, E. N. M., Buermans, H. P. J., Waasdorp, M., Stoorvogel, W., Wauben, M. H. M., and 't Hoen, P. A. C. (2012). Deep sequencing of RNA from immune cell-derived vesicles uncovers the selective incorporation of small non-coding RNA biotypes with potential regulatory functions. Nucleic Acids Res. 40 (18), 9272-9285. doi: 10.1093/nar/gks658

Ohto, U., and Shimizu, T. (2016). Structural aspects of nucleic acid-sensing Tolllike receptors. Biophys. Rev. 8 (1), 33-43. doi: 10.1007/s12551-015-0187-1

Ojo-Amaize, E. A., Salimonu, L. S., Williams, A. I., Akinwolere, O. A., Shabo, R., Alm, G. V., et al. (1981). Positive correlation between degree of parasitemia, interferon titers, and natural killer cell activity in Plasmodium falciparuminfected children. J. Immunol. 127 (6), 2296-2300.

Olivier, M., Van Den Ham, K., Shio, M. T., Kassa, F. A., and Fougeray, S. (2014). Malarial pigment hemozoin and the innate inflammatory response. Front. Immunol. 5:25. doi: 10.3389/fimmu.2014.00025

O’Brien, J. D., Iqbal, Z., Wendler, J., and Amenga-Etego, L. (2016). Inferring Strain Mixture within Clinical Plasmodium falciparum Isolates from Genomic Sequence Data. PloS Comput. Biol. 12 (6), e1004824. doi: 10.1371/journal.pcbi.1004824

O’Neill, L. A., Brown, Z., and Ward, S. G. (2003). Toll-like receptors in the spotlight. Nat. Immunol. 4 (4):299. doi: 10.1038/ni0403-299

Pagola, S., Stephens, P. W., Bohle, D. S., Kosar, A. D., and Madsen, S. K. (2000). The structure of malaria pigment beta-haematin. Nature 404 (6775), 307-310. doi: $10.1038 / 35005132$
Palomo, J., Fauconnier, M., Coquard, L., Gilles, M., Meme, S., Szeremeta, F., et al. (2013). Type I interferons contribute to experimental cerebral malaria development in response to sporozoite or blood-stage Plasmodium berghei ANKA. Eur. J. Immunol. 43 (10), 2683-2695. doi: 10.1002/eji.201343327

Pandey, A. V., and Tekwani, B. L. (1996). Formation of haemozoin/beta-haematin under physiological conditions is not spontaneous. FEBS Lett. 393 (2-3), 189193. doi: 10.1016/0014-5793(96)00881-2

Parmar, R., Patel, H., Yadav, N., Parikh, R., Patel, K., Mohankrishnan, A., et al. (2018). Infectious Sporozoites of Plasmodium berghei Effectively Activate Liver CD8alpha(+) Dendritic Cells. Front. Immunol. 9:192. doi: 10.3389/ fimmu.2018.00192

Parroche, P., Lauw, F. N., Goutagny, N., Latz, E., Monks, B. G., Visintin, A., et al. (2007). Malaria hemozoin is immunologically inert but radically enhances innate responses by presenting malaria DNA to Toll-like receptor 9. Proc. Natl. Acad. Sci. U.S.A. 104 (6), 1919-1924. doi: 10.1073/pnas.0608745104

Pattaradilokrat, S., Li, J., Wu, J., Qi, Y., Eastman, R. T., Zilversmit, M., et al. (2014). Plasmodium genetic loci linked to host cytokine and chemokine responses. Genes Immun. 15 (3), 145-152. doi: 10.1038/gene.2013.74

Penet, M. F., Abou-Hamdan, M., Coltel, N., Cornille, E., Grau, G. E., de Reggi, M., et al. (2008). Protection against cerebral malaria by the low-molecular-weight thiol pantethine. Proc. Natl. Acad. Sci. U. S. A. 105 (4), 1321-1326. doi: $10.1073 /$ pnas.0706867105

Peng, Y. C., Qi, Y., Zhang, C., Yao, X., Wu, J., Pattaradilokrat, S., et al. (2020). Plasmodium yoelii Erythrocyte-Binding-like Protein Modulates Host Cell Membrane Structure, Immunity, and Disease Severity. mBio 11 (1), e02995e02919. doi: 10.1128/mBio.02995-19

Pestka, S., Krause, C. D., and Walter, M. R. (2004). Interferons, interferon-like cytokines, and their receptors. Immunol. Rev. 202, 8-32. doi: 10.1111/j.01052896.2004.00204.x

Pichyangkul, S., Yongvanitchit, K., Kum-Arb, U., Hemmi, H., Akira, S., Krieg, A. M., et al. (2004). Malaria blood stage parasites activate human plasmacytoid dendritic cells and murine dendritic cells through a toll-like receptor 9-dependent pathway. $J$. Immunol. 172 (8), 4926-4933. doi: 10.4049/jimmunol.172.8.4926

Prudencio, M., Rodriguez, A., and Mota, M. M. (2006). The silent path to thousands of merozoites: the Plasmodium liver stage. Nat. Rev. Microbiol. 4 (11), 849-856. doi: 10.1038/nrmicro1529

Radtke, A. J., Kastenmuller, W., Espinosa, D. A., Gerner, M. Y., Tse, S. W., Sinnis, P., et al. (2015). Lymph-node resident CD8alpha+ dendritic cells capture antigens from migratory malaria sporozoites and induce CD8+ T cell responses. PloS Pathog. 11 (2), e1004637. doi: 10.1371/journal.ppat.1004637

Ramirez-Ortiz, Z. G., Lee, C. K., Wang, J. P., Boon, L., Specht, C. A., and Levitz, S. M. (2011). A nonredundant role for plasmacytoid dendritic cells in host defense against the human fungal pathogen Aspergillus fumigatus. Cell Host Microbe 9 (5), 415-424. doi: 10.1016/j.chom.2011.04.007

Ratajczak, J., Miekus, K., Kucia, M., Zhang, J., Reca, R., Dvorak, P., et al. (2006). Embryonic stem cell-derived microvesicles reprogram hematopoietic progenitors: evidence for horizontal transfer of mRNA and protein delivery. Leukemia 20 (5), 847-856. doi: 10.1038/sj.leu.2404132

Ridder, K., Keller, S., Dams, M., Rupp, A. K., Schlaudraff, J., Del Turco, D., et al. (2014). Extracellular vesicle-mediated transfer of genetic information between the hematopoietic system and the brain in response to inflammation. J. Neuroimmunol. 275 (1-2), 165-165. doi: 10.1016/j.jneuroim.2014.08.444

Rocha, B. C., Marques, P. E., Leoratti, F. M. S., Junqueira, C., Pereira, D. B., Antonelli, L., et al. (2015). Type I Interferon Transcriptional Signature in Neutrophils and Low-Density Granulocytes Are Associated with Tissue Damage in Malaria. Cell Rep. 13 (12), 2829-2841. doi: 10.1016/j.celrep.2015.11.055

Ropert, C., and Gazzinelli, R. T. (2000). Signaling of immune system cells by glycosylphosphatidylinositol (GPI) anchor and related structures derived from parasitic protozoa. Curr. Opin. Microbiol. 3 (4), 395-403. doi: 10.1016/s13695274(00)00111-9

Rubartelli, A., and Lotze, M. T. (2007). Inside, outside, upside down: damageassociated molecular-pattern molecules (DAMPs) and redox. Trends Immunol. 28 (10), 429-436. doi: 10.1016/j.it.2007.08.004

Rytel, M. W., Rose, H. D., and Stewart, R. D. (1973). Absence of circulating interferon in patients with malaria and with American trypanosomiasis. Proc. Soc. Exp. Biol. Med. 144 (1), 122-123. doi: 10.3181/00379727-144-37539

Sarasin-Filipowicz, M., Wang, X., Yan, M., Duong, F. H., Poli, V., Hilton, D. J., et al. (2009). Alpha interferon induces long-lasting refractoriness of JAK-STAT 
signaling in the mouse liver through induction of USP18/UBP43. Mol. Cell Biol. 29 (17), 4841-4851. doi: 10.1128/MCB.00224-09

Sasai, M., Linehan, M. M., and Iwasaki, A. (2010). Bifurcation of Toll-like receptor 9 signaling by adaptor protein 3. Science 329 (5998), 1530-1534. doi: 10.1126/ science. 1187029

Schneider, W. M., Chevillotte, M. D., and Rice, C. M. (2014). Interferonstimulated genes: a complex web of host defenses. Annu. Rev. Immunol. 32, 513-545. doi: 10.1146/annurev-immunol-032713-120231

Schofield, L., and Grau, G. E. (2005). Immunological processes in malaria pathogenesis. Nat. Rev. Immunol. 5 (9), 722-735. doi: 10.1038/nri1686

Schofield, L., and Hackett, F. (1993). Signal transduction in host cells by a glycosylphosphatidylinositol toxin of malaria parasites. J. Exp. Med. 177 (1), 145-153. doi: 10.1084/jem.177.1.145

Schwarzer, E., Turrini, F., Ulliers, D., Giribaldi, G., Ginsburg, H., and Arese, P. (1992). Impairment of macrophage functions after ingestion of Plasmodium falciparum-infected erythrocytes or isolated malarial pigment. J. Exp. Med. 176 (4), 1033-1041. doi: 10.1084/jem.176.4.1033

Schwarzer, E., Alessio, M., Ulliers, D., and Arese, P. (1998). Phagocytosis of the malarial pigment, hemozoin, impairs expression of major histocompatibility complex class II antigen, CD54, and CD11c in human monocytes. Infect. Immun. 66 (4), 1601-1606. doi: 10.1128/IAI.66.4.1601-1606.1998

Schwarzer, E., Bellomo, G., Giribaldi, G., Ulliers, D., and Arese, P. (2001). Phagocytosis of malarial pigment haemozoin by human monocytes: a confocal microscopy study. Parasitol 123 (Pt 2), 125-131. doi: 10.1017/ s0031182001008216

Scragg, I. G., Hensmann, M., Bate, C. A., and Kwiatkowski, D. (1999). Early cytokine induction by Plasmodium falciparum is not a classical endotoxin-like process. Eur. J. Immunol. 29 (8), 2636-2644. doi: 10.1002/(SICI)1521-4141 (199908)29:08<2636::AID-IMMU2636>3.0.CO;2-Y

Sebina, I., James, K. R., Soon, M. S., Fogg, L. G., Best, S. E., Labastida Rivera, F., et al. (2016). IFNAR1-Signalling Obstructs ICOS-mediated Humoral Immunity during Non-lethal Blood-Stage Plasmodium Infection. PloS Pathog. 12 (11), e1005999. doi: 10.1371/journal.ppat.1005999

Seth, R. B., Sun, L. J., Ea, C. K., and Chen, Z. J. J. (2005). Identification and characterization of MAVS, a mitochondrial antiviral signaling protein that activates NF-kappa B and IRF3. Cell 122 (5), 669-682. doi: 10.1016/ j.cell.2005.08.012

Shang, G., Zhu, D., Li, N., Zhang, J., Zhu, C., Lu, D., et al. (2012). Crystal structures of STING protein reveal basis for recognition of cyclic di-GMP. Nat. Struct. Mol. Biol. 19 (7), 725-727. doi: 10.1038/nsmb.2332

Sharma, S., DeOliveira, R. B., Kalantari, P., Parroche, P., Goutagny, N., Jiang, Z., et al. (2011). Innate immune recognition of an AT-rich stem-loop DNA motif in the Plasmodium falciparum genome. Immunity 35 (2), 194-207. doi: 10.1016/j.immuni.2011.05.016

Sheehan, K. C., Lazear, H. M., Diamond, M. S., and Schreiber, R. D. (2015). Selective Blockade of Interferon-alpha and -beta Reveals Their NonRedundant Functions in a Mouse Model of West Nile Virus Infection. PloS One 10 (5), e0128636. doi: 10.1371/journal.pone.0128636

Shio, M. T., Eisenbarth, S. C., Savaria, M., Vinet, A. F., Bellemare, M. J., Harder, K. W., et al. (2009). Malarial hemozoin activates the NLRP3 inflammasome through Lyn and Syk kinases. PloS Pathog. 5 (8), e1000559. doi: 10.1371/ journal.ppat.1000559

Silva-Barrios, S., and Stager, S. (2017). Protozoan Parasites and Type I IFNs. Front. Immunol. 8:14. doi: 10.3389/fimmu.2017.00014

Sinha, A., Ignatchenko, V., Ignatchenko, A., Mejia-Guerrero, S., and Kislinger, T. (2014). In-depth proteomic analyses of ovarian cancer cell line exosomes reveals differential enrichment of functional categories compared to the. NCI 60 proteome. Biochem. Biophys. Res. Commun. 445 (4), 694-701. doi: 10.1016/ j.bbrc.2013.12.070

Sisquella, X., Ofir-Birin, Y., Pimentel, M. A., Cheng, L., Abou Karam, P., Sampaio, N. G., et al. (2017). Malaria parasite DNA-harbouring vesicles activate cytosolic immune sensors. Nat. Commun. 8, 1985. doi: 10.1038/s41467-017-02083-1

Skorokhod, O. A., Alessio, M., Mordmuller, B., Arese, P., and Schwarzer, E. (2004). Hemozoin (malarial pigment) inhibits differentiation and maturation of human monocyte-derived dendritic cells: a peroxisome proliferator-activated receptor-gamma-mediated effect. J. Immunol. 173 (6), 4066-4074. doi: 10.4049/ jimmunol.173.6.4066
Spaulding, E., Fooksman, D., Moore, J. M., Saidi, A., Feintuch, C. M., Reizis, B., et al. (2016). STING-Licensed Macrophages Prime Type I IFN Production by Plasmacytoid Dendritic Cells in the Bone Marrow during Severe Plasmodium yoelii Malaria. PloS Pathog. 12 (10), e1005975. doi: 10.1371/ journal.ppat.1005975

Stevenson, M. M., and Riley, E. M. (2004). Innate immunity to malaria. Nat. Rev. Immunol. 4 (3), 169-180. doi: 10.1038/nri1311

Subramaniam, K. S., Spaulding, E., Ivan, E., Mutimura, E., Kim, R. S., Liu, X., et al. (2015). The T-Cell Inhibitory Molecule Butyrophilin-Like 2 Is Up-regulated in Mild Plasmodium falciparum Infection and Is Protective During Experimental Cerebral Malaria. J. Infect. Dis. 212 (8), 1322-1331. doi: 10.1093/infdis/jiv217

Sun, W., Li, Y., Chen, L., Chen, H., You, F., Zhou, X., et al. (2009). ERIS, an endoplasmic reticulum IFN stimulator, activates innate immune signaling through dimerization. Proc. Natl. Acad. Sci. U.S.A. 106 (21), 8653-8658. doi: 10.1073/pnas.0900850106

Sun, L., Wu, J., Du, F., Chen, X., and Chen, Z. J. (2013). Cyclic GMP-AMP synthase is a cytosolic DNA sensor that activates the type I interferon pathway. Science 339 (6121), 786-791. doi: 10.1126/science.1232458

Sweeney, S. S., Hammaker, D., and Firestein, G. S. (2004). Novel functions of the IKK-related kinase IKK in RA: Regulation of c-jun and metalloproteinases. Arthritis Rheum. 50 (9), S654-S654.

Takaoka, A., and Yamada, T. (2019). Regulation of signaling mediated by nucleic acid sensors for innate interferon-mediated responses during viral infection. Int. Immunol. 31 (8), 477-488. doi: 10.1093/intimm/dxz034

Takeda, K., Kaisho, T., and Akira, S. (2003). Toll-like receptors. Annu. Rev. Immunol. 21, 335-376. doi: 10.1146/annurev.immunol.21.120601.141126

Takeuchi, O., and Akira, S. (2010). Pattern Recognition Receptors and Inflammation. Cell 140 (6), 805-820. doi: 10.1016/j.cell.2010.01.022

Tamura, T., Kimura, K., Yui, K., and Yoshida, S. (2015). Reduction of conventional dendritic cells during Plasmodium infection is dependent on activation induced cell death by type I and II interferons. Exp. Parasitol. 159, 127-135. doi: 10.1016/j.exppara.2015.09.010

Tang, D., Kang, R., Coyne, C. B., Zeh, H. J., and Lotze, M. T. (2012). PAMPs and DAMPs: signal 0s that spur autophagy and immunity. Immunol. Rev. 249 (1), 158-175. doi: 10.1111/j.1600-065X.2012.01146.x

Tauro, B. J., Greening, D. W., Mathias, R. A., Ji, H., Mathivanan, S., Scott, A. M., et al. (2012). Comparison of ultracentrifugation, density gradient separation, and immunoaffinity capture methods for isolating human colon cancer cell line LIM1863-derived exosomes. Methods 56 (2), 293-304. doi: 10.1016/ j.ymeth.2012.01.002

Teijaro, J. R., Ng, C., Lee, A. M., Sullivan, B. M., Sheehan, K. C., Welch, M., et al. (2013). Persistent LCMV infection is controlled by blockade of type I interferon signaling. Science 340 (6129), 207-211. doi: 10.1126/science.1235214

Torre, S., Polyak, M. J., Langlais, D., Fodil, N., Kennedy, J. M., Radovanovic, I., et al. (2017). USP15 regulates type I interferon response and is required for pathogenesis of neuroinflammation. Nat. Immunol. 18 (1), 54-63. doi: $10.1038 /$ ni.3581

Tricarico, C., Clancy, J., and D'Souza-Schorey, C. (2017). Biology and biogenesis of shed microvesicles. Small GTPases 8 (4), 220-232. doi: 10.1080/ 21541248.2016.1215283

Uze, G., Schreiber, G., Piehler, J., and Pellegrini, S. (2007). The receptor of the type I interferon family. Curr. Top. Microbiol. Immunol. 316, 71-95. doi: 10.1007/ 978-3-540-71329-6_5

Valadi, H., Ekstrom, K., Bossios, A., Sjostrand, M., Lee, J. J., and Lotvall, J. O. (2007). Exosome-mediated transfer of mRNAs and microRNAs is a novel mechanism of genetic exchange between cells. Nat. Cell Biol. 9 (6), 654-U672. doi: $10.1038 /$ ncb1596

Vaughan, A. M., Sack, B. K., Dankwa, D., Minkah, N., Nguyen, T., Cardamone, H., et al. (2018). A Plasmodium Parasite with Complete Late Liver Stage Arrest Protects against Preerythrocytic and Erythrocytic Stage Infection in Mice. Infect. Immun. 86 (5), e00088-18. doi: 10.1128/IAI.00088-18

Venereau, E., Ceriotti, C., and Bianchi, M. E. (2015). DAMPs from Cell Death to New Life. Front. Immunol. 6:422. doi: 10.3389/fimmu.2015.00422

Vigario, A. M., Belnoue, E., Cumano, A., Marussig, M., Miltgen, F., Landau, I., et al. (2001). Inhibition of Plasmodium yoelii blood-stage malaria by interferon alpha through the inhibition of the production of its target cell, the reticulocyte. Blood 97 (12), 3966-3971. doi: 10.1182/blood.v97.12.3966 
Vigario, A. M., Belnoue, E., Gruner, A. C., Mauduit, M., Kayibanda, M., Deschemin, J. C., et al. (2007). Recombinant human IFN-alpha inhibits cerebral malaria and reduces parasite burden in mice. J. Immunol. 178 (10), 6416-6425. doi: 10.4049/jimmunol.178.10.6416

Vijay, K. (2018). Toll-like receptors in immunity and inflammatory diseases: Past, present, and future. Int. Immunopharmacol. 59, 391-412. doi: 10.1016/ j.intimp.2018.03.002

Voisine, C., Mastelic, B., Sponaas, A. M., and Langhorne, J. (2010). Classical $\mathrm{CD} 11 \mathrm{c}+$ dendritic cells, not plasmacytoid dendritic cells, induce $\mathrm{T}$ cell responses to Plasmodium chabaudi malaria. Int. J. Parasitol. 40 (6), 711-719. doi: 10.1016/j.ijpara.2009.11.005

von der Weid, T., Kitamura, D., Rajewsky, K., and Langhorne, J. (1994). A dual role for B cells in Plasmodium chabaudi chabaudi (AS) infection? Res. Immunol. 145 (6), 412-419. doi: 10.1016/s0923-2494(94)80170-3

Williams, C., Rodriguez-Barrueco, R., Silva, J. M., Zhang, W. J., Hearn, S., Elemento, O., et al. (2014). Double-stranded DNA in exosomes: a novel biomarker in cancer detection. Cell Res. 24 (6), 766-769. doi: 10.1038/ cr.2014.44

Wilson, E. B., Yamada, D. H., Elsaesser, H., Herskovitz, J., Deng, J., Cheng, G., et al. (2013). Blockade of chronic type I interferon signaling to control persistent LCMV infection. Science 340 (6129), 202-207. doi: 10.1126/ science. 1235208

Wu, J., and Chen, Z. J. (2014). Innate immune sensing and signaling of cytosolic nucleic acids. Annu. Rev. Immunol. 32, 461-488. doi: 10.1146/annurevimmunol-032713-120156

Wu, X. Z., Gowda, N. M., Kumar, S., and Gowda, D. C. (2010). Protein-DNA Complex Is the Exclusive Malaria Parasite Component That Activates Dendritic Cells and Triggers Innate Immune Responses. J. Immunol. 184 (8), 4338-4348. doi: 10.4049/jimmunol.0903824

Wu, J., Sun, L., Chen, X., Du, F., Shi, H., Chen, C., et al. (2013). Cyclic GMP-AMP is an endogenous second messenger in innate immune signaling by cytosolic DNA. Science 339 (6121), 826-830. doi: 10.1126/science.1229963

Wu, J., Tian, L., Yu, X., Pattaradilokrat, S., Li, J., Wang, M., et al. (2014). Strainspecific innate immune signaling pathways determine malaria parasitemia dynamics and host mortality. Proc. Natl. Acad. Sci. U.S.A. 111 (4), E511-E520. doi: 10.1073/pnas.1316467111

Wu, J., Cai, B., Sun, W., Huang, R., Liu, X., Lin, M., et al. (2015a). Genome-wide Analysis of Host-Plasmodium yoelii Interactions Reveals Regulators of the Type I Interferon Response. Cell Rep. 12 (4), 661-672. doi: 10.1016/ j.celrep.2015.06.058

Wu, X., Gowda, N. M., and Gowda, D. C. (2015b). Phagosomal Acidification Prevents Macrophage Inflammatory Cytokine Production to Malaria, and Dendritic Cells Are the Major Source at the Early Stages of Infection: Implication for malaria protective immunity development. J. Biol. Chem. 290 (38), 23135-23147. doi: 10.1074/jbc.M115.671065

Wu, J., Xia, L., Yao, X., Yu, X., Tumas, K. C., Sun, W., et al. (2020). The E3 ubiquitin ligase MARCH1 regulates antimalaria immunity through interferon signaling and T cell activation. Proc. Natl. Acad. Sci. U.S.A. 117 (28), 16567-16578. doi: 10.1073/ pnas.2004332117

Wykes, M. N., and Good, M. F. (2008). What really happens to dendritic cells during malaria? Nat. Rev. Microbiol. 6 (11), 864-870. doi: 10.1038/ nrmicro1988

Xia, L., Wu, J., Pattaradilokrat, S., Tumas, K., He, X., Peng, Y. C., et al. (2018). Detection of host pathways universally inhibited after Plasmodium yoelii infection for immune intervention. Sci. Rep. 8 (1), 15280. doi: 10.1038/ s41598-018-33599-1

Xu, H., Hodder, A. N., Yan, H., Crewther, P. E., Anders, R. F., and Good, M. F. (2000). CD4+ T cells acting independently of antibody contribute to protective immunity to Plasmodium chabaudi infection after apical membrane antigen 1 immunization. J. Immunol. 165 (1), 389-396. doi: 10.4049/jimmunol.165.1.389
Xu, L. G., Wang, Y. Y., Han, K. J., Li, L. Y., Zhai, Z. H., and Shu, H. B. (2005). VISA is an adapter protein required for virus-triggered IFN-beta signaling. Mol. Cell 19 (6), 727-740. doi: 10.1016/j.molcel.2005.08.014

Yao, X., Wu, J., Lin, M., Sun, W., He, X., Gowda, C., et al. (2016). Increased CD40 Expression Enhances Early STING-Mediated Type I Interferon Response and Host Survival in a Rodent Malaria Model. PloS Pathog. 12 (10), e1005930. doi: 10.1371/journal.ppat.1005930

Ye, W. J., Chew, M., Hou, J., Lai, F., Leopold, S. J., Loo, H. L., et al. (2018). Microvesicles from malaria-infected red blood cells activate natural killer cells via MDA5 pathway. PloS Pathog. 14 (10), e1007298. doi: 10.1371/ journal.ppat.1007298

Yoneyama, M., Kikuchi, M., Matsumoto, K., Imaizumi, T., Miyagishi, M., Taira, K., et al. (2005). Shared and unique functions of the DExD/H-box helicases RIG-I, MDA5, and LGP2 in antiviral innate immunity. J. Immunol. 175 (5), 2851-2858. doi: 10.4049/jimmunol.175.5.2851

Yoshimura, A., Naka, T., and Kubo, M. (2007). SOCS proteins, cytokine signalling and immune regulation. Nat. Rev. Immunol. 7 (6), 454-465. doi: 10.1038/ nri2093

Yu, X., Cai, B., Wang, M., Tan, P., Ding, X., Wu, J., et al. (2016). Cross-Regulation of Two Type I Interferon Signaling Pathways in Plasmacytoid Dendritic Cells Controls Anti-malaria Immunity and Host Mortality. Immunity 45 (5), 1093 1107. doi: 10.1016/j.immuni.2016.10.001

Yu, X., Du, Y., Cai, C., Cai, B., Zhu, M., Xing, C., et al. (2018). Inflammasome activation negatively regulates MyD88-IRF7 type I IFN signaling and anti-malaria immunity. Nat. Commun. 9 (1), 4964. doi: 10.1038/s41467-018-07384-7

Zander, R. A., Guthmiller, J. J., Graham, A. C., Pope, R. L., Burke, B. E., Carr, D. J., et al. (2016). Type I Interferons Induce T Regulatory 1 Responses and Restrict Humoral Immunity during Experimental Malaria. PloS Pathog. 12 (10), e1005945. doi: 10.1371/journal.ppat.1005945

Zheng, Y., and Gao, C. (2020). Fine-tuning of antiviral innate immunity by ubiquitination. Adv. Immunol. 145, 95-128. doi: 10.1016/bs.ai.2019.11.004

Zhong, B., Yang, Y., Li, S., Wang, Y. Y., Li, Y., Diao, F., et al. (2008). The adaptor protein MITA links virus-sensing receptors to IRF3 transcription factor activation. Immunity 29 (4), 538-550. doi: 10.1016/j.immuni.2008.09.003

Zhu, J., Krishnegowda, G., and Gowda, D. C. (2005). Induction of proinflammatory responses in macrophages by the glycosylphosphatidylinositols of Plasmodium falciparum: the requirement of extracellular signal-regulated kinase, p38, c-Jun Nterminal kinase and NF-kappaB pathways for the expression of proinflammatory cytokines and nitric oxide. J. Biol. Chem. 280 (9), 8617-8627. doi: 10.1074/ jbc.M413539200

Zhu, J. Z., Wu, X. Z., Goel, S., Gowda, N. M., Kumar, S., Krishnegowda, G., et al. (2009). MAPK-activated Protein Kinase 2 Differentially Regulates Plasmodium falciparum Glycosylphosphatidylinositol-induced Production of Tumor Necrosis Factor-alpha and Interleukin-12 in Macrophages. J. Biol. Chem. 284 (23), 15750-15761. doi: 10.1074/jbc.M901111200

Zhu, S. J., Hendry, J. A., Almagro-Garcia, J., Pearson, R. D., Amato, R., Miles, A., et al. (2019). The origins and relatedness structure of mixed infections vary with local prevalence of P. falciparum malaria. Elife 8, e40845. doi: 10.7554/ eLife.40845

Conflict of Interest: The authors declare that the research was conducted in the absence of any commercial or financial relationships that could be construed as a potential conflict of interest.

Copyright (c) $2020 \mathrm{He}, \mathrm{Xia}, \mathrm{Tumas}, \mathrm{Wu}$ and Su. This is an open-access article distributed under the terms of the Creative Commons Attribution License (CC BY). The use, distribution or reproduction in other forums is permitted, provided the original author(s) and the copyright owner(s) are credited and that the original publication in this journal is cited, in accordance with accepted academic practice. No use, distribution or reproduction is permitted which does not comply with these terms. 